\title{
Obvious Dominance and Random Priority
}

\author{
Marek Pycia and Peter Troyan *
}

May 2018

\begin{abstract}
We characterize the full class of obviously strategy-proof mechanisms in environments without transfers as clinch-or-pass games that we call millipede games. Some millipede games are simple and widely used in practice, while others may be complex, requiring agents to perform lengthy backward induction, and are rarely observed. We introduce a natural strengthening of obvious strategy-proofness called strong obvious strategy-proofness, which eliminates these complex millipede games. We use our definition to characterize the well-known Random Priority mechanism as the unique mechanism that is efficient, fair, and simple to play, thereby explaining its popularity in practical applications.
\end{abstract}

\section{Introduction}

Consider a group of agents who must come together to make a choice from some set of potential outcomes that will affect each of them. This can be modeled as having the agents play a "game", taking turns choosing from sets of actions (possibly simultaneously), with the final outcome determined by the decisions made by all of the agents each time they were called to play. To ensure that the ultimate decision taken satisfies desirable normative properties (e.g., efficiency), the incentives given to the agents are crucial. The standard route taken in mechanism design to ensure good incentives is to appeal to the revelation principle and look for direct mechanisms that are strategy-proof, i.e., mechanisms where agents are

${ }^{*}$ Pycia: UCLA and U. Zurich; Troyan: University of Virginia. First presentation: April 2016. First posted draft: June 2016. For their comments, we would like to thank Itai Ashlagi, Eduardo Azevedo, Ben Golub, Yannai Gonczarowski, Ed Green, Fuhito Kojima, Shengwu Li, Giorgio Martini, Stephen Morris, Nick Netzer, Erling Skancke, Utku Unver, the Eco 514 students at Princeton, and the audiences at the NBER Market Design workshop, NEGT'16, NC State, ITAM, NSF/CEME Decentralization, the Econometric Society Meetings, and UBC. Simon Lazarus provided excellent research assistance. Pycia would like to acknowledge the financial support of the William S. Dietrich II Economic Theory Center at Princeton. 
simply asked to report their private information, and it is always in their interest to do so truthfully, no matter what the other agents do. However, this is useful only to the extent the participants understand that a given mechanism is strategy-proof, and indeed, there is evidence many real-world agents do not tell the truth, even in strategy-proof mechanisms. In other words, strategy-proof mechanisms, while theoretically appealing, may not actually be easy for participants to play in practice. What mechanisms, then, are actually "simple to play"? And further, what simple mechanisms satisfy other normatively desirable properties such as efficiency and fairness? This paper provides answers to these questions.

We begin by constructing the full class of obviously strategy-proof (Li, 2017), or OSP, mechanisms in general social choice environments without transfers. Social choice problems without transfers are ubiquitous in the real-world, and examples include refugee resettlement, school choice, organ exchange, public housing allocation, course allocation, and voting, among others. ${ }^{1}$ We call the class of OSP games in these environments millipede games (for reasons that will become clear shortly). While some millipede games, such as sequential dictatorships, are frequently encountered and are indeed simple to play, others are rarely observed in market-design practice, and their strategy-proofness is not necessarily immediately clear. In particular, some millipede games may still require agents to look far into the future and to perform potentially complicated backward induction reasoning. Thus, to further delineate the class of mechanism that are simple to play, we introduce a new criterion called strong obvious strategy-proofness (SOSP). We construct the full class of strongly obviously strategy-proof games and show that strong obvious strategy-proofness selects the subset of millipede games that are observed in practice while eliminating the more complex millipede games that are rarely (if ever) used. Combining SOSP with standard efficiency and fairness axioms narrows the space of mechanisms down even further to a unique choice, and one that is commonly observed in practice: Random Priority.

An imperfect-information extensive-form game is obviously strategy-proof if, whenever an agent is called to play, there is an action such that even the worst possible final outcome from following the given action is at least as good as the best possible outcome from taking any other action at the node in question, where the best and worst cases are determined by fixing the agent's strategy and considering all possible strategies that could be played by her opponents in the future. Li (2017) provides both a theoretical behavioral foundation for OSP games being "simple to play" and experimental evidence that, in certain settings, OSP mechanisms do indeed lead to higher rates of truth-telling in practice than their counterparts

\footnotetext{
${ }^{1}$ See e.g. Roth (2015) and Jones and Teytelboym (2016) for resettlement, Abdulkadiroğlu and Sönmez (2003) for school choice, Roth, Sönmez, and Ünver (2004) for transplants, Sönmez and Ünver (2010) and Budish and Cantillon (2012) for course allocation, and Arrow (1963) for voting and social choice.
} 
that are strategy-proof, but not obviously so. ${ }^{2}$

Obvious strategy-proofness restricts the set of potential games from which a designer may choose, which raises the question of exactly which games are obviously strategy-proof. Our first main result, Theorem 1, constructs the full class of OSP mechanisms as the class of millipede games. In a millipede game, Nature moves first and chooses a deterministic subgame, after which agents engage in a game of passing and clinching that resembles the well-studied centipede game (Rosenthal, 1981). To describe this deterministic subgame in this introduction, for expositional ease, we focus on allocation problems with agents who demand at most one unit; the argument for more general social choice environments is similar. An agent is presented with some subset of objects that she can 'clinch', or, take immediately and leave the game; she also may be given the opportunity to 'pass', and remain in the game. ${ }^{3}$ If this agent passes, another agent is presented with an analogous choice. Agents keep passing among themselves until one of them clinches some object. When an agent clinches an object, this is her last move and she leaves the game.

The class of millipede games includes many games that are commonly seen in practice, such as Random Priority and Serial Dictatorships, which are millipede games in which the agent who moves can always clinch any object that is still available. However, our characterization shows that these are not the only mechanisms that are obviously strategy-proof. Some millipede games require substantial foresight on the part of the agents, similar to the foresight required in centipede games. For instance, it is possible to construct a millipede game such that a player is offered the possibility of clinching his second-choice object, but not his top choice object, even though it is still available. If the agent passes, he might not be given the opportunity to clinch any of his top fifty objects in the next one hundred moves. The obviously dominant strategy requires the agent to pass, even though he may never be offered his top choice for some plays of the other agents. (We provide an example of such a game in Figure 2.) Recognizing that passing is obviously dominant requires the agent to perform lengthy backward induction.

As another illustration of the backward-induction requirements inherent in some obviously dominant strategies, consider chess. If White can always force a win, then any winning strategy of White is obviously dominant, yet the strategic choices in chess are far from obvious; indeed, to the best of our knowledge even the question whether White actually has

\footnotetext{
${ }^{2} \mathrm{Li}$ also shows that OSP games are those that can be implemented under bilateral commitment between the designer and the agents.

${ }^{3}$ In general, we may allow this agent to clinch the same object in several ways; while the choice between them has no impact on the agent's outcome, it might affect the allocation of others. Additionally, there need not be a passing action that allows the agent to stay in the game, but if there is, there can be at most one such action, in order to preserve obvious strategy-proofness.
} 
a winning strategy remains open. Obvious dominance allows for agents who are unable to contingently reason about their opponents' actions, but presumes that they do have perfect foresight about their own future selves. Thus, if White can force a win, then, at each node, he is able to determine the obviously dominant move by reasoning backwards from all future nodes at which he is called to play. ${ }^{4}$

These examples suggest that some obviously dominant strategies may be hard to identify, which poses the question of what properties of an extensive-form game guarantee that the game is simple to play. To address this question, we introduce a refinement of obvious strategy-proofness, which we call strong obvious strategy-proofness. An extensive-form game is strongly obviously strategy-proof if, whenever an agent is called to play, there is an action such that even the worst possible final outcome from that action is at least as good as the best possible outcome from any other action, where what is possible may depend on all future actions, including actions by the agent's future-self. Thus, strongly obviously dominant strategies are those that are weakly better than all alternative strategies even if the agent is concerned that she might tremble in the future or has time-inconsistent preferences. $^{5}$

Indeed, all SOSP games can be implemented without requiring agents to look far into the future and perform lengthy backwards induction. We show this by proving that strong obvious strategy-proofness eliminates the complex members of the more general class of millipede games, and that strongly obviously strategy-proof games take the form of curated dictatorships. In curated dictatorships, all agents move only once and (with the possible exception of the penultimate mover) their outcomes do not depend on the choices of agents who move after them. The optimal play thus relies on the agents's ability to see at most one step ahead in the game. ${ }^{6}$

While most of our focus is on incentives and simplicity, there are other important criteria when designing a mechanism, such as efficiency and fairness. Our final result shows when these desiderata are added as necessary requirements, only one mechanism survives: Random Priority. Random Priority has a long history and is extensively used in a wide variety of practical allocation problems. School choice, worker assignment, course allocation, and the allocation of public housing are just a few of many examples, both formal and informal. Random Priority is well-known to have good efficiency, fairness, and incentive properties. ${ }^{7}$ However, it has until now remained unknown whether there are other such mechanisms,

\footnotetext{
${ }^{4}$ We would like to thank Eduardo Azevedo and Ben Golub for raising this point.

${ }^{5}$ For rich explorations of agents with time-inconsistent preferences, see e.g., Laibson (1997), Gul and Pesendorfer $(2001 ; 2004)$, Jehiel $(1995 ; 2001)$; for bounded horizon backward induction see also Ke (2015).

${ }^{6}$ In particular, strongly obviously strategy-proof and Pareto efficient games take the simple form of almostsequential dictatorships, studied earlier in a different context by Pycia and Ünver (2016).

${ }^{7}$ For discussion of efficiency and fairness see, e.g., Abdulkadiroğlu and Sönmez (1998), Bogomolnaia and Moulin (2001), and Che and Kojima (2010).
} 
and if so, what explains the relative popularity of RP over these alternatives. ${ }^{8}$ Theorem 6 provide answers to these questions by showing that there is no other such mechanism: a game is strongly obviously strategy-proof, Pareto efficient, and treats agents equally (a standard, and relatively weak, fairness axiom) if and only if it is Random Priority. This insight resolves positively the quest to establish Random Priority as the unique mechanism with good incentive, efficiency, and fairness properties, thereby explaining its popularity in practical market design settings.

Our results build on the key contributions of Li (2017), who formalized obvious strategyproofness and established its desirability as an incentive property by both providing a theoretical foundation and experimental evidence that participants play their dominant strategy more often in obviously strategy-proof mechanisms (e.g., ascending auctions, dynamic Random Priority) than in mechanisms that are strategy-proof, but not obviously so (sealed-bid auctions, static Random Priority). ${ }^{9}$ While Li looks at specific mechanisms in the no-transfer setting, we characterize the entire class of obviously strategy-proof mechanisms, and provide an explanation for the popularity of Random Priority over all other mechanisms, results which have no counterpart in his work. Finally, our analysis of strong obvious strategyproofness furthers our understanding of why some extensive forms of a mechanism are more often encountered in practice, despite being both equivalent according to the standard Myerson-Riley revelation principle and obviously strategy-proof.

Following up on Li's work, but preceding ours, Ashlagi and Gonczarowski (2016) show that stable mechanisms such as Deferred Acceptance are not obviously strategy-proof, except in very restrictive environments where Deferred Acceptance simplifies to an obviously strategy-proof game with a 'clinch or pass' structure similar to simple millipede games (though they do not describe it in these terms). Other related papers include Troyan (2016), who studies obviously strategy-proof allocation via the popular Top Trading Cycles (TTC) mechanism, and provides a characterization of the priority structures under which TTC is

\footnotetext{
${ }^{8}$ In single-unit demand allocation with at most three agents and three objects, Bogomolnaia and Moulin (2001) proved that Random Priority is the unique mechanism that is strategy-proof, efficient, and symmetric. In markets in which each object is represented by many copies, Liu and Pycia (2011) and Pycia (2011) proved that Random Priority is the asymptotically unique mechanism that is symmetric, asymptotically strategyproof, and asymptotically ordinally efficient. While these earlier results looked at either very small or very large markets, ours is the first characterization that holds for any number of agents and objects.

${ }^{9} \mathrm{Li}$ also shows that the classic top trading cycles (TTC) mechanism of Shapley and Scarf (1974), in which each agent starts by owning exactly one object, is not obviously strategy-proof. Troyan (2016) expands this by considering more general ownership structures, and shows that TTC is obviously strategy-proof if and only if at any time, at most two agents own all of the available objects. Also of note is Loertscher and Marx (2015) who study environments with transfers and construct a prior-free obviously strategy-proof mechanism that becomes asymptotically optimal as the number of buyers and sellers grows.
} 
OSP-implementable. Following our work, Arribillaga et al. (2017) characterize the voting rules that are obviously strategy-proof on the domain of single-peaked preferences and, in an additional result, in environments with two alternatives. In contrast to our work, they focus on characterizing social choice functions instead of games. ${ }^{10}$ Zhang and Levin (2017a; 2017b) provide decision-theoretic foundations for obvious dominance and explore weaker incentive concepts.

More generally, this paper adds to our understanding of incentives, efficiency, and fairness in settings without transfers. In addition to Gibbard (1973, 1977) and Satterthwaite (1975), and the allocation papers mentioned above, the literature on mechanisms satisfying these key objectives includes Pápai (2000), Ehlers (2002) and Pycia and Unver (2017; 2016) who characterized efficient and group strategy-proof mechanisms in settings with single-unit demand, and Pápai (2001) and Hatfield (2009) who provided such characterizations for settings with multi-unit demand. ${ }^{11}$ Liu and Pycia (2011), Pycia (2011), Morrill (2014), Hakimov and Kesten (2014), Ehlers and Morrill (2017), and Troyan et al. (2018) characterize mechanisms that satisfy certain incentive, efficiency, and fairness objectives.

\section{Model}

Let $\mathcal{N}=\left\{i_{1}, \ldots, i_{N}\right\}$ be a set of agents, and $\mathcal{X}$ a finite set of outcomes. ${ }^{12}$ Each agent has a preference ranking over outcomes, where we write $x \succsim_{i} y$ to denote that $x$ is weakly preferred to $y$. We allow for indifferences, and write $x \sim_{i} y$ if $x \succsim_{i} y$ and $y \succsim_{i} x$. The domain of preferences of agent $i \in \mathcal{N}$ is denoted $\mathcal{P}_{i}$, where each $\mathcal{P}_{i}$ determines a partition over the set of outcomes such that, for all $x, y \in \mathcal{X}$ that belong to the same element of the partition, we have $x \sim_{i} y$ for all $\succsim_{i} \in \mathcal{P}_{i}$. Each preference relation of agent $i$ may then be associated with the corresponding strict ranking of the elements of the partition. We will generally work with this strict ranking, denoted $\succ_{i}$, and will sometimes refer to $\succ_{i}$ as an agent's type.

The main assumption we make on the preference domains is that they are rich, in the following sense: given a partition, every strict ranking of the elements of the partition is in

\footnotetext{
${ }^{10}$ Other papers that follow on our work include: Bade and Gonczarowski (2017), who study obviously strategy-proof and efficient social choice rules in several environments, and Mackenzie (2017), who introduces the notion of a "round table mechanism" for OSP implementation and draws parallels with the standard Myerson-Riley revelation principle for direct mechanisms.

${ }^{11}$ Pycia and Ünver (2016) characterized individually strategy-proof and Arrovian efficient mechanisms. For an analysis of these issues under additional feasibility constraints, see also Dur and Ünver (2015).

${ }^{12}$ The assumption that $\mathcal{X}$ is finite simplifies the exposition and it is satisfied in such examples of our setting as voting and the no-transfer allocation environments listed in the introduction. This assumption can be relaxed. For instance, our analysis goes through with no substantive changes if we allow infinite $\mathcal{X}$ endowed with a topology such that agents' preferences are continuous in this topology and the relevant sets of outcomes are compact.
} 
$\mathcal{P}_{i}$. This assumption is satisfied for a wide variety of preference structures. ${ }^{13}$ Two important examples are the canonical voting environment (where every agent can strictly rank all alternatives) and allocating a set of indivisible goods (where each agent cares only about the set of goods she receives). In the former case, each agent partitions $\mathcal{X}$ into $|\mathcal{X}|$ singleton subsets and an agent's type $\succ_{i}$ is a strict preference relation over $\mathcal{X}$. In the latter case, an outcome $x \in \mathcal{X}$ describes the entire allocation to each of the agents. Since agents are indifferent over how objects she does not receive are assigned to others, each element of agent $i$ 's partition of $\mathcal{X}$ can be identified with her own allocation, and her type $\succ_{i}$ is then a (strict) ranking of these allocations. Where this assumption fails is in settings with transfers, in which it is assumed that each agent always prefers having more money to less.

When dealing with lotteries, we are agnostic as to how agents evaluate them, as long as the following property holds: an agent prefers lottery $\mu$ over $\nu$ if for any outcomes $x \in \operatorname{supp}(\mu)$ and $y \in \operatorname{supp}(\nu)$ this agent weakly prefers $x$ over $y$; the preference between $\mu$ and $\nu$ is strict if, additionally, at least one of the preferences between $x \in \operatorname{supp}(\mu)$ and $y \in \operatorname{supp}(\nu)$ is strict. This mild assumption is satisfied for expected utility agents; it is also satisfied for agents who prefer $\mu$ to $\nu$ as soon as $\mu$ first-order stochastically dominates $\nu$.

To determine the outcome that will be implemented, the planner designs a game $\Gamma$ for the agents to play. Formally, we consider imperfect-information, extensive-form games with perfect recall, which are defined in the standard way: there is a finite collection of partially ordered histories (sequences of moves), $\mathcal{H}$. At every non-terminal history $h \in \mathcal{H}$, one agent is called to play and has a finite set of actions $A(h)$ from which to choose. We allow for random moves by Nature. We use the notation $\omega(h)$ for a realization of Nature's move at history $h$ and $\omega:=(\omega(h))_{\{h \in \mathcal{H} \text { :nature moves at } h\}}$ to denote a realization of Nature's moves throughout the game at every history at which Nature is called to play. Each terminal history is associated with an outcome in $\mathcal{X}$, and agents receive payoffs at each terminal history that are consistent with their preferences over outcomes $\succ_{i}$.

We use the notation $h^{\prime} \subseteq h$ to denote that $h^{\prime}$ is a subhistory of $h$ (equivalently, $h$ is a continuation history of $h^{\prime}$ ), and write $h \subset h^{\prime}$ when $h \subseteq h^{\prime}$ but $h \neq h^{\prime} . \mathcal{H}_{i}(h)$ denotes the set of (strict) subhistories $h^{\prime} \subset h$ at which agent $i$ is called to move. When useful, we sometimes write $h^{\prime}=(h, a)$ to denote the history $h^{\prime}$ that is reached by starting at history $h$ and following the action $a \in A(h)$.

An information set $\mathcal{I}$ of agent $i$ is a set of histories such that for any $h, h^{\prime} \in \mathcal{I}$ and any subhistories $\tilde{h} \subseteq h$ and $\tilde{h}^{\prime} \subseteq h^{\prime}$ at which $i$ moves at least one of the following two symmetric conditions obtains: either (i) there is a history $\tilde{h}^{*} \subseteq \tilde{h}$ such that $\tilde{h}^{*}$ and $\tilde{h}^{\prime}$ are in the same information set, $A\left(\tilde{h}^{*}\right)=A\left(\tilde{h}^{\prime}\right)$, and $i$ makes the same move at $\tilde{h}^{*}$ and $\tilde{h}^{\prime}$, or (ii) there is a

\footnotetext{
${ }^{13}$ In Appendix B, we slightly relax this assumption.
} 
history $\tilde{h}^{*} \subseteq \tilde{h}^{\prime}$ such that $\tilde{h}^{*}$ and $\tilde{h}$ are in the same information set, $A\left(\tilde{h}^{*}\right)=A(\tilde{h})$, and $i$ makes the same move at $\tilde{h}^{*}$ and $\tilde{h}$. We denote by $\mathcal{I}(h)$ the information set containing history $h{ }^{14}$ These imperfect information games allow us to incorporate incomplete information in the standard way in which Nature moves first and determines agents' types.

A strategy for a player $i$ in game $\Gamma$ is a function $S_{i}$ that specifies an action at each one of her information sets. ${ }^{15}$ When we want to refer to the strategies of different types $\succ_{i}$ of agent $i$, we write $S_{i}\left(\succ_{i}\right)$ for the strategy followed by agent $i$ for whom Nature drew type $\succ_{i}$; in particular, $S_{i}\left(\succ_{i}\right)(\mathcal{I})$ denotes the action chosen by agent $i$ with type $\succ_{i}$ at information set $\mathcal{I}$. We use $S_{\mathcal{N}}\left(\succ_{\mathcal{N}}\right)=\left(S_{i}\left(\succ_{i}\right)\right)_{i \in \mathcal{N}}$ to denote the strategy profile for all of the agents when the type profile is $\succ_{\mathcal{N}}=\left(\succ_{i}\right)_{i \in \mathcal{N}}$. An extensive-form mechanism, or simply a mechanism, is an extensive-form game $\Gamma$ together with a profile of strategies $S_{\mathcal{N}}$. Two extensive-form mechanisms $\left(\Gamma, S_{\mathcal{N}}\right)$ and $\left(\Gamma^{\prime}, S_{\mathcal{N}}^{\prime}\right)$ are equivalent if for every profile of types $\succ_{\mathcal{N}}=\left(\succ_{i}\right)_{i \in \mathcal{N}}$, the resulting distribution over outcomes when agents follow $S_{\mathcal{N}}\left(\succ_{\mathcal{N}}\right)$ in $\Gamma$ is the same as when agents follow $S_{\mathcal{N}}^{\prime}\left(\succ_{\mathcal{N}}\right)$ in $\Gamma^{\prime}{ }^{16}$

Remark 1. In the sequel we establish several equivalences. Each of them is an equivalence of two mechanism which also satisfy additional criteria such as obvious strategy-proofness or strong obvious strategy-proofness. We can thus formally strengthen these results to $\mathcal{C}$ equivalence defined as follows. A solution concept $\mathcal{C}(\cdot)$ maps any game $\Gamma$ into a subset of strategy profiles $\mathcal{C}(\Gamma)$. The interpretation is that the strategy profiles in $\mathcal{C}(\Gamma)$ are those profiles that satisfy the solution concept $\mathcal{C}$; for example, if we were concerned with strategyproof implementation $(\mathcal{C}=\mathrm{SP})$, then $\mathrm{SP}(\Gamma)$ would be all strategy profiles $S_{\mathcal{N}}$ such that $S_{i}\left(\succ_{i}\right)$ is a weakly dominant strategy for all $i$ and all types $\succ_{i}$ in game $\Gamma$. Two extensiveform mechanisms $\left(\Gamma, S_{\mathcal{N}}\right)$ and $\left(\Gamma^{\prime}, S_{\mathcal{N}}^{\prime}\right)$ are $\mathcal{C}$-equivalent if the following two conditions are satisfied: (i) for every profile of types $\succ_{\mathcal{N}}=\left(\succ_{i}\right)_{i \in \mathcal{N}}$, the resulting distribution over outcomes when agents play $S_{\mathcal{N}}\left(\succ_{\mathcal{N}}\right)$ in $\Gamma$ is the same as when agents play $S_{\mathcal{N}}^{\prime}\left(\succ_{\mathcal{N}}\right)$ in $\Gamma^{\prime}$ and (ii) $S_{\mathcal{N}}\left(\succ_{\mathcal{N}}\right) \in \mathcal{C}(\Gamma)$ and $S_{\mathcal{N}}^{\prime}\left(\succ_{\mathcal{N}}\right) \in \mathcal{C}\left(\Gamma^{\prime}\right)$. In other words, two mechanisms are $\mathcal{C}$-equivalent if they are equivalent and the strategies satisfy the solution concept $\mathcal{C}$ in the respective games.

\footnotetext{
${ }^{14}$ We will see shortly that it is without loss of generality to assume all information sets are singletons, and so will be able to drop the $\mathcal{I}(h)$ notation and identify each information set with the unique sequence of actions (i.e., history) taken to reach it.

${ }^{15}$ We consider pure strategies, but the analysis can be easily extended to mixed strategies.

${ }^{16}$ The equivalence concept here is outcome-based, and hence different from the procedural equivalence concept of Kohlberg and Mertens (1986).
} 


\section{Millipede Games}

In this section, we begin our analysis of which games are "simple to play" by characterizing the entire class of games that are obviously strategy-proof. Following Li (2017), given a game $\Gamma$, a strategy $S_{i}$ obviously dominates another strategy $S_{i}^{\prime}$ for player $i$ if, starting from any earliest information set $\mathcal{I}$ at which these two strategies diverge, ${ }^{17}$ the worst possible payoff to the agent from playing $S_{i}$ is at least as good as the best possible payoff from $S_{i}^{\prime}$, where the best/worst case outcomes are determined over all possible $\left(S_{-i}, \omega\right)$. A profile of strategies $S_{\mathcal{N}}(\cdot)=\left(S_{i}(\cdot)\right)_{i \in \mathcal{N}}$ is obviously dominant if for every player $i$ and every type $\succ_{i}$, the strategy $S_{i}\left(\succ_{i}\right)$ obviously dominates any other strategy. When there exists a profile of strategies $S_{\mathcal{N}}(\cdot)$ that is obviously dominant, we say $\Gamma$ is obviously strategy-proof (OSP).

Obvious strategy-proofness is one way of capturing what it means for a mechanism to be simple to play. Li (2017) provides a theoretical foundation for this intuition by showing that obviously dominant strategies are those that can be recognized as dominant by cognitively limited agents who are unable to engage in contingent reasoning. ${ }^{18}$ Further, he provides empirical evidence that, for certain mechanisms, participants are more likely to tell the truth under extensive-form implementations (which are OSP) than under the corresponding normal form (which are strategy-proof, but not obviously so). ${ }^{19}$

If obvious strategy-proofness as a solution concept does indeed capture what games are simple to play, then an important question is to determine precisely which games satisfy this criterion. Our first main result is to characterize the entire class of obviously strategyproof mechanisms as a class of games that we call "millipede games". Intuitively, a millipede game is a take-or-pass game similar to a centipede game, but with more players and more actions (i.e., "legs") at each node. Figure 1 shows the extensive form of a millipede game for the special case of object allocation with single-unit demand, where the agents are labeled $i, j, k, \ldots$ and the objects are labeled $w, x, y, \ldots$ At the start of the game, the first mover, agent $i$ has three options: he can take $x$, take $y$, or pass to agent $j .{ }^{20}$ If he takes an object,

\footnotetext{
${ }^{17}$ That is, information set $\mathcal{I}$ is on the path of play under both $S_{i}$ and $S_{i}^{\prime}$ and both strategies choose the same action at all earlier information sets but choose a different action at $\mathcal{I}$. Li (2017) refers to such an information set as an earliest point of departure. Note that for two strategies, there will in general be multiple earliest points of departure.

${ }^{18}$ See also Zhang and Levin (2017b) who provide further decision-theoretic foundations for obvious strategy-proofness.

${ }^{19}$ For example, people are more likely to tell the truth in the extensive-form of the Random Priority mechanism than in the corresponding normal form (where they are asked to submit an entire list of preferences to 'the mechanism' ex-ante). From the standpoint of the classical revelation principle, these two mechanisms should be strategically equivalent.

${ }^{20}$ In the general definition of a millipede game, it will be possible that none of the actions are passing actions and so all actions are taking actions. The key restriction imposed by obvious strategy-proofness is that if there is a passing action, there can only be one. A formal definition is given below.
} 


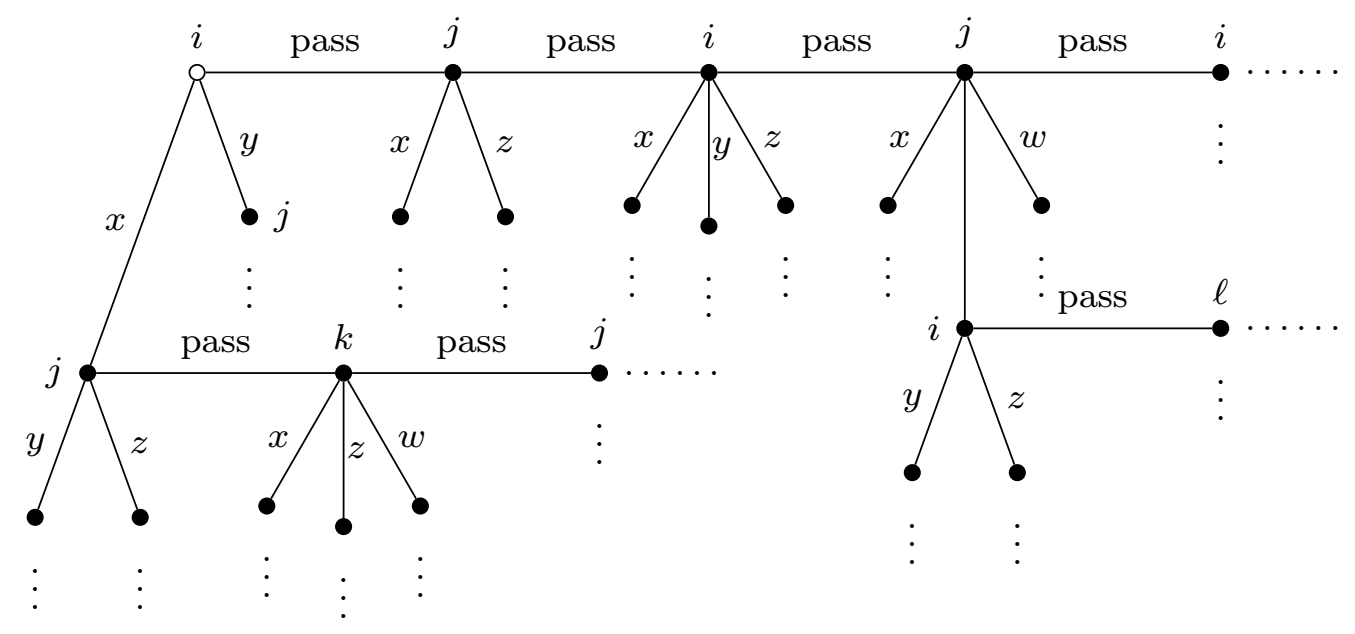

Figure 1: An example of a millipede game in the context of object allocation.

he leaves the game and it continues with a new agent. If he passes, then agent $j$ can take $x$, take $z$, or pass back to $i$. If he passes back to $i$, then $i$ 's possible choices increase from his previous move (he can now take $z$ ). The game continues in this manner until all objects have been allocated.

While Figure 1 considers an object allocation environment, millipede games can be defined more generally on any preference structure that satisfies our assumptions of Section 2. Recall that each agent's preference domain $\mathcal{P}_{i}$ partitions the outcome space $\mathcal{X}$ into indifference classes. We use the term payoff to refer to the indifference class associated with a particular element of the partition. We say that a payoff $x$ is possible for agent $i$ at history $h$ if there is a strategy profile of all the agents (including choices made by Nature) such that $h$ is on the path of the game and, under this strategy profile, agent $i$ obtains payoff $x$ (i.e., the outcome that obtains under the given strategy profile is in the indifference class that gives her payoff $x$ ). For any history $h, P_{i}(h)$ denotes the set of payoffs that are possible for $i$ at $h$. We say agent $i$ has clinched payoff $x$ at history $h$ if agent $i$ receives payoff $x$ at all terminal histories $\bar{h} \supseteq h$. If after following action $a \in A(h)$, an agent receives the same payoff for every terminal $\bar{h} \supseteq(h, a)$, we say that $a$ is a clinching action. Clinching actions are generalizations of the "taking actions" of Figure 1 to environments where the outcomes/payoff structure may be different from object allocation (where more generally, what $i$ is clinching is a particular indifference class for herself). We denote the set of payoffs that $i$ can clinch at history $h$ by $C_{i}(h) .{ }^{21}$ If an action $a \in A(h)$ is not a clinching action, then it is called a passing action.

\footnotetext{
${ }^{21}$ That is, $x \in C_{i}(h)$ if there is some action $a \in A(h)$ such that $i$ receives payoff $x$ for all terminal $\bar{h} \supseteq(h, a)$. At a terminal history $\bar{h}$, no agent is called to move and there are no actions; however, for the purposes of constructing millipede games below, it will be useful to define $C_{i}(\bar{h})=\{x\}$ for all $i$, where $x$ is the payoff
} 
A millipede game is a finite extensive-form game of perfect information that satisfies the following properties: Nature either moves once, at the empty history $\emptyset$, or Nature has no moves. At any history $h$ at which an agent, say $i$, moves, all but at most one action are clinching actions; the remaining action (if there is one) is a passing action. ${ }^{22}$ And finally, for all $i$, all histories $h$ at which $i$ moves and all terminal histories, and all payoffs $x$, at least one of the following holds: ${ }^{23}$ (a) $x \in P_{i}(h)$; or (b) $x \notin P_{i}(\tilde{h})$ for some $\tilde{h} \in \mathcal{H}_{i}(h)$; or (c) $x \in \cup_{\tilde{h} \in \mathcal{H}_{i}(h)} C_{i}(\tilde{h})$; or $(\mathrm{d}) \cup_{\tilde{h} \in \mathcal{H}_{i}(h)} C_{i}(\tilde{h}) \subseteq C_{i}(h)$. In words, these last conditions require that, for every payoff $x$ that was at some point possible for $i$, either: (a) $x$ remains possible at $h$, or (b) it was not possible already at an earlier history, or (c) it was clinchable at an earlier history, or (d) each payoff clinchable at an earlier history is clinchable at $h$.

Conditions (a)-(d) ensure that, if an agent's top still-possible outcome is not clinchable at some history $h$, then she is able to guarantee that the payoff she ultimately receives is at least as good as any payoff she could have clinched at $h$, which is necessary for passing to be obviously dominant. To see this, consider an agent whose top payoff is $x$, and a history $h$ such that (a), (b) and (c) fail for $x$. This means at all prior moves, $x$ was possible (by "not (b)"), but not clinchable (by "not (c)"), and so obvious dominance requires $i$ to pass. However, $x$ has disappeared as a possibility at $h$ (by "not (a)"), and so to ensure that $i$ does not regret her choice to pass at an earlier history, at $h$, we must offer her the opportunity to clinch anything she could have clinched previously, which is condition (d).

Notice that millipede games have a recursive structure: the continuation game that follows any action is also a millipede game. A simple example of a millipede game is a deterministic serial dictatorship in which no agent ever passes and there is only one active agent at each node. ${ }^{24} \mathrm{~A}$ more complex example is given in Figure $1 .{ }^{25}$

Our first main result is to characterize the class of OSP games and mechanisms as the class of millipede games with greedy strategies. A strategy is called greedy if at each move at which the agent can clinch the best still-possible outcome for her, the strategy has the agent clinch this outcome; otherwise, the agent passes.

Theorem 1. Every obviously strategy-proof mechanism $\left(\Gamma, S_{\mathcal{N}}\right)$ is equivalent to a millipede game with the greedy strategy. Every millipede game with the greedy strategy is obviously

associated with the unique outcome that obtains at terminal history $\bar{h}$.

${ }^{22}$ There may be several clinching actions associated with the same final payoff.

${ }^{23}$ Recall that $\mathcal{H}_{i}(h)=\left\{h^{\prime} \subsetneq h: i\right.$ moves at $\left.h^{\prime}\right\}$.

${ }^{24} \mathrm{An}$ agent is active at history $h$ of a millipede game if the agent moves at $h$, or the agent moved prior to history $h$ and has not yet clinched an outcome.

${ }^{25}$ The first more complex example of a millipede game we know of is due to Ashlagi and Gonczarowski (2016). They construct an example of OSP-implementation of deferred acceptance on some restricted preference domains. On these restricted domains, DA reduces to a millipede game (though they do not classify the actions as "passing" or "clinching" actions). Later work by Bade and Gonczarowski (2017) gives an example of a millipede game that, while shorter, is, in some respects, even more complex than our examples. 
strategy-proof.

This theorem is applicable in many environments. This includes allocation problems in which agents care only about the object(s) they receive, in which case, clinching actions correspond to taking a specified object and leaving the remaining objects to be distributed amongst the remaining agents. Theorem 1 also applies to standard social choice problems in which no agent is indifferent between any two outcomes (e.g., voting), in which case clinching corresponds to determining the final outcome for all agents. In such environments, Theorem 1 implies that each OSP game is equivalent to a game in which either there are only two outcomes that are possible when the first agent moves (and the first mover can either clinch any of them, or can clinch one of them or pass to a second agent, who is presented with an analogous choice, etc.), or the first agent to move can clinch any possible outcome and has no passing action. The latter case is the standard dictatorship, with a possible restricted set of possible outcomes, while the former case resembles the almost-sequential dictatorships we study in the next section.

We now outline the main ideas of the proof of Theorem 1. First, to show that greedy strategies are obviously dominant in a millipede game, note that if, at some history $h$, an agent can clinch her top still-possible outcome, it is clearly obviously dominant to do so. Harder is to show that if an agent cannot clinch her top still-possible outcome at $h$, then passing is obviously dominant. Formally, this follows from conditions (a)-(d) above. The complete technical details can be found in the appendix.

The more difficult (and interesting) part of Theorem 1 is the first part: for any OSP game $\Gamma$, we can find an equivalent millipede game with greedy strategies. The proof in the appendix breaks the argument down into three main steps.

Step 1. Every OSP game $\Gamma$ is equivalent to a perfect information OSP game $\Gamma^{\prime}$ in which Nature moves once, as the first mover.

Intuitively, this follows because if we break any information set with imperfect information to several different information sets with perfect information, the set of outcomes that are possible shrinks. For an action $a$ to be obviously dominant, the worst possible outcome from $a$ must be (weakly) better than the best possible outcome from any other $a^{\prime}$. If the set of possibilities shrinks, then the worst case from $a$ only improves, and the best case from $a^{\prime}$ worsens; thus, if a was obviously dominant in $\Gamma$, it will remain so in $\Gamma^{\prime} .^{26}$

Step 2. At every history, all actions except for possibly one are clinching actions.

\footnotetext{
${ }^{26}$ That every OSP game is equivalent to an OSP game with perfect information was first pointed out in a footnote by Ashlagi and Gonczarowski (2016). The same footnote also states that de-randomizing an OSP game leads to an OSP game. For completeness, the appendix contains the (straightforward) proofs of these statements.
} 


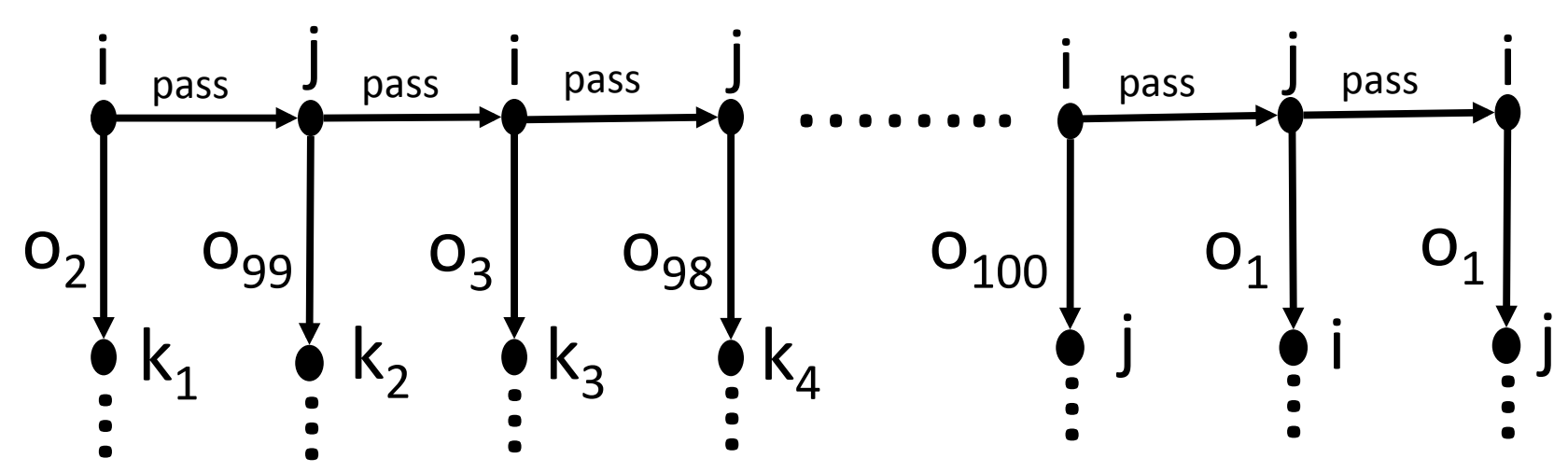

Figure 2: An example of a millipede game.

Step 2 allows us to greatly simplify the class of OSP games to "clinch or pass" games. Indeed, if there were two passing actions $a$ and $a^{\prime}$ at some history $h$, then following each of $a$ and $a^{\prime}$ there are at least two outcomes that are possible for $i$. There will always be a type of agent $i$ for which one of the possibilities following $a$ is at best his second choice, while one of the possibilities following $a^{\prime}$ is his first choice. This implies that $i$ must have some strategy that can guarantee himself this first-choice outcome in the continuation game following $a^{\prime}$ (by OSP). We can then construct an equivalent game in which $i$ is able to clinch this first-choice outcome already at $h$. Proceeding in this way, we are able to eliminate one of the actions $a$ or $a^{\prime}$.

Step 3. If agent $i$ passes at a history $h$, then the payoff she ultimately receives must be at least as good as any of the payoffs she could have clinched at $h$.

An agent may follow the passing action if she cannot clinch her favorite possible outcome today, and so she passes, hoping she will be able to move again in the future and get it then. To retain obvious strategy-proofness, the game needs to promise agent $i$ that she can never be made worse off by passing. This implies that one of the conditions (a)-(d) in definition above must obtain. Combining steps 1-3 imply that any OSP game $\Gamma$ is equivalent to a millipede game.

Theorem 1 characterizes the entire class of obviously strategy-proof games. We have already briefly mentioned some familiar dictatorship-like games that fit into this class (e.g., Random Priority, also known as Random Serial Dictatorship, in an object allocation environment). Another example of a millipede game is given in Figure 2. Here, there are 100 agents $\left\{i, j, k_{1}, \ldots, k_{98}\right\}$ and 100 objects $\left\{o_{1}, o_{2}, \ldots, o_{100}\right\}$ to be assigned. The game begins with agent $i$ being offered the opportunity to clinch $o_{2}$, or pass to $j$. Agent $j$ can then either clinch $o_{99}$, in which case the next mover is $k_{2}$, or pass back to $i$, and so on. Now, consider the type of agent $i$ that prefers the objects in the order of their index: $o_{1} \succ_{i} O_{2} \succ_{i} \cdots \succ_{i} O_{100}$. 
At the very first move of the game, $i$ is offered her second-favorite object, $o_{2}$, even though her top choice, $o_{1}$, is still available. The obviously dominant strategy here requires $i$ to pass. However, if she passes, she may not be offered the opportunity to clinch her top object(s) for hundreds of moves. Further, when considering all of the possible moves of the other agents, if $i$ passes, the game has the potential to go off into thousands of different directions, and in many of them, she will never be able to clinch better than $o_{2}$. Thus, while passing is formally obviously dominant, fully comprehending this still requires the ability to reason far into the future of the game and perform lengthy backwards induction.

\section{Strong Obvious Strategy-Proofness}

The upshot of the previous section is that some OSP mechanisms, such as Random Priority, are indeed quite simple to play; however, the full class of millipede games is much larger, and contains OSP mechanisms that may be quite complex to actually play. The reason is that OSP relaxes the assumption that agents fully comprehend how the choices of other agents will translate into outcomes, but it still presumes that they understand how their own future actions affect outcomes. Thus, while OSP guarantees that when taking an action, agents do not have to reason carefully about what their opponents will do, it still may require that they reason carefully about the continuation game, in particular with regard to their own "future self". Here, we introduce a strengthening of obvious dominance that we call strong obvious dominance.

Definition 1. For an agent $i$ with preferences $\succ_{i}$, strategy $S_{i}$ strongly obviously dominates strategy $S_{i}^{\prime}$ in game $\Gamma$ if, starting at any earliest point of departure $\mathcal{I}$ between $S_{i}$ and $S_{i}^{\prime}$, the best possible outcome from following $S_{i}^{\prime}$ at $\mathcal{I}$ is weakly worse than the worst possible outcome from following $S_{i}$ at $\mathcal{I}$, where the best and worst cases are determined by considering any future play by other agents (including Nature) and agent $i$. If a strategy $S_{i}$ strongly obviously dominates all other $S_{i}^{\prime}$, then we say that $S_{i}$ is strongly obviously dominant.

If a mechanism admits a profile of strongly obviously dominant strategies, we say that it is strongly obviously strategy-proof (SOSP). Returning to the examples at the end of the previous section, Random Priority is SOSP, but the millipede game depicted in Figure 2 is not. Thus, SOSP mechanisms further delineate the class of games that are simple to play, by eliminating the more complex millipede games that may require significant forward-looking behavior and backward induction. ${ }^{27}$

\footnotetext{
${ }^{27}$ Recall also the example of chess discussed in the introduction: if White can force a win, then any winning strategy of White is obviously dominant, yet the strategic choices in chess seem far from obvious. Chess will not admit a strongly obviously dominant strategy.
} 
Strong obvious strategy-proofness has several appealing features that capture the idea of a game being simple to play. As mentioned above, SOSP strengthens OSP by looking at the worst/best case outcomes for $i$ over all possible future actions that could be taken by $i$ 's opponents and agent $i$ herself. Thus, a strongly obviously dominant strategy is one that is weakly better than all alternative strategies even if the agent is concerned that she might tremble in the future or has time-inconsistent preferences. Also, as we will see shortly, SOSP games can be implemented so that each agent is called to move at most once.

The next two results formalize the previous discussion. We first show that strongly obviously dominant strategies are those that are robust to agents' potential misunderstandings about the game they are playing. ${ }^{28}$ Consider an agent who, when she is called to play at some history, knows the outcomes that are possible from each action, but may not know precisely how those outcomes depend on the future play. Strongly obviously dominant strategies are those that remain weakly dominant for such agents. To state these results, we consider perfect-information games for which no histories are off the path of play. ${ }^{29}$ We say that an extensive-form game $\Gamma$ is outcome-set equivalent to extensive-form game $\Gamma^{\prime}$ if there is a bijection $\phi$ between histories such that for all $h$, the set of possible outcomes following history $h$ in $\Gamma$ is equivalent to the set of possible outcomes following history $\phi(h)$ in $\Gamma^{\prime}$. We then obtain the following. ${ }^{30}$

Theorem 2. For all $i$ and $\succ_{i}$, strategy $S_{i}$ is strongly obviously dominant in $\Gamma$ if and only if in all outcome-set equivalent games $\Gamma^{\prime}$, the corresponding strategy $S_{i}^{\prime}$ is weakly dominant.

Another implication of the definition of strongly obviously dominant strategies that highlights their simplicity is that in any SOSP game, each agent can have at most one history at which her choice of action is payoff-relevant. Formally, we say a history $h$ at which agent $i$ moves is payoff-irrelevant for this agent if either (i) there is only one action at $h$ or (ii) $i$ receives the same payoff at all terminal histories $\bar{h} \supset h$; if $i$ moves at $h$ and this history is not payoff-irrelevant, then it is payoff-relevant for $i$. The definition of SOSP and richness of the preference domain give us the following.

Theorem 3. Along each path of a SOSP game, there is at most one payoff-relevant history for each agent.

\footnotetext{
${ }^{28} \mathrm{Li}$ (2017) shows a similar result for OSP. He also shows that OSP mechanisms are precisely the mechanisms that can be implemented with bilateral commitment; this result does not extend to our setting. The reason is that bilateral commitment presumes that agents are perfectly forward looking and do not make errors in single-agent games, and SOSP relaxes this assumption.

${ }^{29} \mathrm{Li}$ (2017) refers to this latter restriction as a pruned game. He shows that pruning a game by removing all histories that are never reached for any type profile preserves obvious dominance; the same is true for strong obvious dominance. A formal definition is given in the appendix.

${ }^{30}$ We note also that this result continues to hold even if our richness assumption on preferences is violated.
} 
This result allows us to further conclude that the unique payoff-relevant history (if it exists) is the first history at which an agent chooses from among two or more actions. While an agent might be called to act later in the game, and her choice might influence the continuation game and the payoffs for other agents, it cannot affect her own payoff.

Just as Theorem 1 did for OSP, we can provide a simple canonical form for all SOSP mechanisms. Strengthening OSP to SOSP eliminates the complex examples of millipede games, such as those in Figure 2. We say a game $\Gamma$ is a curated dictatorship if is a perfectinformation game in which Nature moves first (if at all). The agents then move sequentially, with each agent called to play at most once. The ordering of the agents and the sets of possible outcomes at each history are determined by Nature's action and the actions taken by earlier agents. As long as there are either at least three payoffs possible payoffs for an agent when he is called to move or there is exactly one such payoff, the agent can clinch any of the possible payoffs, while at the same time also selecting a message from a pre-determined set of messages. ${ }^{31}$ When exactly two payoffs are possible for the agent who moves, the agent can be faced with either a choice between them (clinching and picking an accompanying message), or, he might be given a possibility to clinch one of these payoffs (and picking an accompanying message) and passing (with no message). ${ }^{32}$

Theorem 4. Every strongly obviously strategy-proof mechanism $\left(\Gamma, S_{\mathcal{N}}\right)$ is equivalent to a curated dictatorship with the greedy strategy. Every curated dictatorship with the greedy strategy is strongly obviously strategy-proof.

That curated dictatorships are SOSP is immediate. To get a sense for why the the other part of the theorem is true let us restrict attention to millipede games that are SOSP (the complete proof is in the appendix). Because we are constructing an equivalent game, we can assume that at no history an agent has exactly one action. Given the definition of a curated dictatorship, it is sufficient to show that pruned millipede games in which there is a history $h$ at which the acting agent has three or more possible payoffs and a passing move are not SOSP. Consider such a game and let $h$ be such a history. By Theorem $3, h$ is first time $i$ is called to act, and so $h$ is on the path of play for all types of agent $i$. Because there can be at most one passing action, at least one payoff must be clinchable for $i$, say $x$,

\footnotetext{
${ }^{31}$ As discussed in Section 3, (S)OSP games may present an agent with several different ways to clinch the same payoff; sending a message is a simple way to encode which of the clinching actions the agent takes. Fixing a clinched payoff, each possible message may affect the future of the game, e.g., by determining who the next mover is, though the agent's own payoff is not affected.

${ }^{32}$ Notice that at histories with three or more possible payoffs for the moving agent, curated dictatorships can be interpreted in terms of (personalized) posted prices: the agent is given a menu of choices and can freely select one of them. In fact, we can partially relax the richness assumption by allowing transfers, and a posted-price analogue of Theorem 4 would remain true.
} 
and at least one must not be clinchable at $h$, say $y$ (if everything were clinchable, then the passing action can be pruned). Let $z \neq x, y$ be some third payoff that is possible at $h$. To complete the argument, note that either agent $i$ of type $y \succ_{i} x \succ_{i} z \succ_{i} \cdots$ or agent $i$ of type $y \succ_{i} z \succ_{i} x \succ_{i} \cdots$ has no strongly obviously dominant action at $h$, and so the game is not SOSP.

\section{Random Priority}

Thus far, all of our results have been about incentives and what makes a game "simple to play". While incentives are undoubtedly important, they are not the only consideration when designing a mechanism; efficiency and fairness are two other key goals. As an application of our new definition of SOSP, we can use it, combined with very natural fairness and efficiency axioms, to characterize the popular Random Priority (RP) mechanism.

While we have informally been using object allocation as a running example for our previous results, we now make this more concrete. There is a set of agents $\mathcal{N}$ and objects $\mathcal{O}$; each agent is to be assigned exactly one of objects, and we assume $|\mathcal{N}|=|\mathcal{O}|$. In this context, an "outcome" is an allocation of objects to agents, and the outcome space $\mathcal{X}$ is the space of all such allocations. Agents care only about their own assignment, and are indifferent between allocations where the assignment of others may vary, but their own assignment is unchanged. We consider single-unit demand for simplicity and comparison with the prior literature that often focuses on this case (Abdulkadiroğlu and Sönmez, 1998; Bogomolnaia and Moulin, 2001; Che and Kojima, 2009; Liu and Pycia, 2011), but equivalent results hold in more general models that lie in the framework laid out in Section 2.

We begin by considering only incentives and efficiency, and characterize SOSP and efficient mechanisms as the class of almost-sequential dictatorships. ${ }^{33}$ An almost-sequential dictatorship is a perfect-information game in which nature moves first and then the agents move in turn, with each agent moving at most once. At his move, an agent picks an object and sends a message. As long as there are at least three objects unallocated, the moving agent can choose from all still-available objects. When there are two objects remaining, an agent can be faced with either a choice between them, or, he might be given a choice between one of these objects for sure or giving the next agent an opportunity to allocate the remaining two objects among the two of them.

The difference between a serial dictatorship and a sequential dictatorship is that in a serial

\footnotetext{
${ }^{33}$ Pycia and Ünver (2016) use the same name for deterministic mechanisms without messages that belong to the class we study; they show that these are exactly the deterministic mechanisms which are strategy-proof and Arrovian efficient with respect to a complete social welfare function. We use the name their introduced because our class is a natural extension of theirs.
} 
dictatorship, the ordering of the agents is fixed after the nature's move, while in a sequential dictatorship, the next agent to move may depend on the choices of the earlier agents. The reason we call the mechanism described above an "almost" sequential dictatorship is because it works exactly as a sequential dictatorship if there are three or more objects remaining, with a small modification allowed when only two objects remain.

Theorem 5. Every strongly obviously strategy-proof and Pareto efficient mechanism $\left(\Gamma, S_{\mathcal{N}}\right)$ is equivalent to an almost-sequential dictatorship with the greedy strategy. Every almostsequential dictatorship with the greedy strategy is strongly obviously strategy-proof and Pareto efficient.

That almost-sequential dictatorships are SOSP and efficient is immediate. The proof of the other direction follows easily from Theorem 4. Given any SOSP and efficient game $\Gamma$, the proof of Theorem 4 shows how to construct an equivalent curated dictatorship $\Gamma^{\prime}$. By efficiency and the richness of the preference domain, all objects are possible for the first mover in $\Gamma^{\prime}$. Therefore, by definition of a curated dictatorship, the first mover is offered the opportunity to clinch any object immediately, so long as $|\mathcal{O}| \geq 3$. Similarly, efficiency and richness imply that the second mover is offered the opportunity to clinch any stillremaining object, so long as there are at least 3 or more objects remaining to be allocated. The same applies to the third, fourth, etc. movers until exactly two objects remain. When two objects, say $\{x, y\}$, remain, efficiency still implies that both objects are possible for the penultimate mover. If this agent is offered the opportunity to clinch either, the game is a serial dictatorship, which is a special case of an almost-sequential dictatorship. Alternatively, he may be given a choice between clinching one of $x$ or $y$, say $x$, or passing and allowing the final mover to allocate $x$ and $y$ between them. In either case, it is easy to see that the overall game $\Gamma^{\prime}$ is an almost-sequential dictatorship. ${ }^{34}$

Almost-sequential dictatorships are SOSP and efficient, but they are not necessarily fair. Indeed, consider a simple serial dictatorship with a fixed ordering of the agents (which is a special case of an almost-sequential dictatorship). Then, the first agent in the ordering, $i_{1}$, always gets his first choice, while the last agent, $i_{N}$, always gets whatever remains. Formally, a mechanism satisfies equal treatment of equals if, whenever two agents have the same type, they receive the same distribution over payoffs. It is easy to see that the above serial dictatorship fails this criterion. The natural way to resolve this unfairness is to order the agents randomly, and allow them to pick in this order, which gives the popular Random Priority (RP) mechanism. It is simple to check that RP is SOSP, efficient, and satisfies equal treatment of equals. The next result says that RP is in fact the only such mechanism.

\footnotetext{
${ }^{34}$ The natural analogue of this theorem remains valid beyond object allocation, for all rich preference domains, with no substantive changes in the proof.
} 
Theorem 6. Every mechanism that is strongly obviously strategy-proof, Pareto efficient, and satisfies equal treatment of equals is equivalent to Random Priority with greedy strategies. Furthermore, Random Priority with greedy strategies is strongly obviously strategy-proof, Pareto efficient, and satisfies equal treatment of equals.

Random Priority succeeds on all three important dimensions: it is simple to play, Pareto efficient, and fair. ${ }^{35}$ However, this is only a partial explanation of its success, as to now, it has remained unknown whether there exist other such mechanisms, and, if so, what explains the relative popularity of RP over these alternatives. ${ }^{36}$ Theorem 6 provides an answer to this question: not only does RP have good efficiency, fairness, and incentive properties, it is indeed the only mechanism that does so, thus explaining the widespread popularity of RP in practice.

\section{Conclusion}

In general social choice environments without transfers, we study the question of what makes a game "simple to play". We begin by characterizing the entire class of obviously strategyproof games, and show that they take the form of clinch-or-pass games that we call millipede games. In a millipede game, each time an agent is called to move, she is offered (potentially several) outcomes that she can clinch immediately and leave the game; she may also in addition be offered the opportunity to pass (and stay in the game). While obviously strategyproof, some millipede games may still require extensive foresight and backwards induction, and so may not necessarily be simple to play for real-world agents.

We thus propose a new definition of simplicity, strong obvious strategy-proofness. Strongly obviously strategy-proof mechanisms eliminate the need for significant foresight and backwards induction: we prove that in these mechanisms each agent is asked to make at most one payoff-relevant choice. While a constraint on the choice of mechanisms, strong obvious strategy-proofness is still weak enough to allow positive results, and indeed admits mechanisms that are seen extensively in practice. In the main no-transfer setting that we consider, we show that SOSP mechanisms are curated dictatorships, a class which includes the Random Priority mechanism. ${ }^{37}$ We also prove a natural characterization of this popular

\footnotetext{
${ }^{35}$ At the cost of substantial strengthening the fairness/symmetry axiom, we could relax SOSP to OSP in Theorem 6.

${ }^{36}$ Bogomolnaia and Moulin (2001) provide a characterization of RP in the special case of $|\mathcal{N}|=3$, but their result does not extend to larger markets; Liu and Pycia (2011) provide a characterization using asymptotic versions of standard axioms in replica economies as the market size grows to infinity.

${ }^{37}$ Also with transfers, some of the most popular mechanisms - namely posted prices - are SOSP. For instance, while eBay began as an auction website, Einav et al. (2018) document a dramatic shift in the 2000s
} 
mechanism by showing that Random Priority is the unique mechanism that is SOSP, Pareto efficient, and satisfies equal treatment of equals. In other words, our results show that Random Priority is the unique mechanism satisfying these desirable incentive, efficiency, and fairness properties, providing an explanation for its widespread use.

\section{A Proofs}

\section{A.1 Proof of Theorem 1}

Before proceeding with the proof of Theorem 1, we first define the concepts of possible, guaranteeable, and clinchable outcomes/actions more formally.

\section{A.1.1 Definitions}

Fix a game $\Gamma$. Let $S=\left(S_{i}\right)_{i \in \mathcal{N}}$ denote a strategy profile for the agents, and recall that $\omega$ denotes one particular realization of Nature's moves (i.e., at each $h$ at which nature is called to play). Define $z(h, S, \omega) \in \mathcal{X}$ as the unique final outcome reached when play starts at some history $h$ and proceeds according to $(S, \omega)$.

We first discuss the distinction between types of payoffs (possible vs. guaranteeable) and then the distinction between types of actions (clinching actions vs. passing actions). Recall that agents may be indifferent between several outcomes. For any outcome $x \in \mathcal{X}$, let $[x]_{i}=\left\{y \in \mathcal{X}: y \sim_{i} x\right\}$ denote the $x$-indifference class of agent $i$, and define

$$
X_{i}\left(h, S_{i}\right)=\left\{[x]_{i}: z\left(h,\left(S_{i}, S_{-i}\right), \omega\right) \in[x]_{i} \text { for some }\left(S_{-i}, \omega\right)\right\}
$$

to be the possible indifference classes that may obtain for agent $i$ starting at history $h$ if she follows strategy $S_{i}$. Consider an agent $i$ of type $\succ_{i}$. If there exists some $S_{i}$ such that $[x]_{i} \in X_{i}\left(h, S_{i}\right)$, then we then we say that $[x]_{i}$ is possible for $i$ at $h$. If, further, there exists some $S_{i}$ such that $X_{i}\left(h, S_{i}\right)=\left\{[x]_{i}\right\}$, then we say $[x]_{i}$ is guaranteeable for $i$ at $h$. Let

$$
\begin{gathered}
P_{i}(h)=\left\{[x]_{i}: \exists S_{i} \text { s.t. }[x]_{i} \in X_{i}\left(h, S_{i}\right)\right\} \\
G_{i}(h)=\left\{[x]_{i}: \exists S_{i} \text { s.t. } X_{i}\left(h, S_{i}\right)=\left\{[x]_{i}\right\}\right\}
\end{gathered}
$$

from auctions to posted prices as the predominant selling mechanism on the platform. Our work thus provides an additional reason why posted prices are so widely used in practice. For an earlier microfoundation of posted prices, see Hagerty and Rogerson (1987). Armstrong (1996) shows that posted prices (combined with bundling) can achieve good revenues (for other analyses of revenues of posted price mechanisms see also e.g. Chawla et al. (2010) and Feldman et al. (2014)). 
be the sets of payoffs that are possible and guaranteeable at $h$, respectively. ${ }^{38}$ Note that $G_{i}(h) \subseteq P_{i}(h)$, and the set $P_{i}(h) \backslash G_{i}(h)$ is the set of payoffs that are possible at $h$, but are not guaranteeable at $h$. (In the proof of the theorem below, we will generally drop the bracket notation $[x]_{i}$ and, when there is no confusion, simply refer to the "payoff $x$ ". Statements such as " $x$ is a possible payoff at $h$ " or " $x \in P_{i}(h)$ " are understood as "some outcome in the indifference class $[x]_{i}$ is possible at $h "$.)

Last, we define a distinction between two kinds of actions: clinching actions and passing actions. Let $i$ be the agent who is to act at a history $h$. Using our notational convention that $(h, a)$ denotes the history obtained by starting at $h$ and following action $a$, the set $P_{i}((h, a))$ is the set of payoffs that would be possible for $i$ if she were to follow action $a$ at $h$. If $P_{i}((h, a))=\left\{[x]_{i}\right\}$, then we say that action $a \in A(h)$ clinches payoff $x$ for $i$. If an action $a$ clinches $x$ for $i$, we call $a$ a clinching action. Note that there can be more than one action that clinches the same payoff $x$ for $i$, though different choices may lead to different payoffs for other agents. Any action of an agent that is not a clinching action is called a passing action. We let $C_{i}(h)$ denote the set of payoffs that are clinchable for $i$ at $h$; that is,

$$
C_{i}(h)=\left\{[x]_{i}: \exists a \in A(h) \text { s.t. } P_{i}((h, a))=\left\{[x]_{i}\right\}\right\}
$$

Note that this definition of $C_{i}(h)$ presumes that agent $i$ is called to play at history $h$. If $\bar{h}$ is a terminal history, then no agent is called to play and there are no actions. However, it will be useful in what follows to define $C_{i}(\bar{h})=\left\{[x]_{i}\right\}$ for all $i$, where $x$ is the unique outcome associated with the terminal history $\bar{h}$.

\section{A.1.2 Proof}

With the above definitions in hand, we can prove Theorem 1 . We start by proving that millipede games are OSP (Proposition 1), and then prove that every OSP game is equivalent to a millipede game (Proposition 2).

Proposition 1. Millipede games with greedy strategies are obviously strategy-proof.

Proof. Let $\Gamma$ be a millipede game. Recall that the greedy strategy for any agent $i$ is defined as follows: for any history $h$ at which $i$ movies, if $i$ can clinch her top payoff in $P_{i}(h)$, then $S_{i}\left(\succ_{i}\right)(h)$ instructs $i$ to follow an action that clinches this payoff; otherwise, $i$ passes at $h .{ }^{39}$

\footnotetext{
${ }^{38}$ Note that $P_{i}(h)$ and $G_{i}(h)$ are well-defined even if $i$ is not the agent who moves at $h$.

${ }^{39}$ There may be multiple ways for $i$ to clinch the same payoff $x$ at $h$, and further, $x$ may in principle still be possible/guaranteeable if $i$ passes at $h$. Our goal is simply to prove the existence of at least one obviously dominant strategy for $i$.
} 
We now show that it is obviously dominant for all agents to follow a greedy strategy. Consider some profile of greedy strategies $\left(S_{i}(\cdot)\right)_{i \in \mathcal{N}}$. For any subset of outcomes $X^{\prime} \subset \mathcal{X}$, define $\operatorname{Top}\left(\succ_{i}, X^{\prime}\right)$ as the best possible payoff in the set $X^{\prime}$ according to preferences $\succ_{i}$, i.e., $x \in \operatorname{Top}\left(\succ_{i}, X^{\prime}\right)$ if and only if $x \succsim_{i} y$ for all $y \in X^{\prime}$ (note that we use our standard convention whereby a payoff $x$ represents the entire indifference class to which $x$ belongs, and so $\operatorname{Top}\left(\succ_{i}, X^{\prime}\right)$ is effectively unique). Then, $\operatorname{Top}\left(\succ_{i}, P_{i}(h)\right)$ denotes $i$ 's top payoff among all payoffs that are possible at history $h$, and $\operatorname{Top}\left(\succ_{i}, C_{i}(h)\right)$ denotes $i$ 's top payoff among all of his clinchable payoffs at $h$. It is clear that if $\operatorname{Top}\left(\succ_{i}, C_{i}(h)\right)=\operatorname{Top}\left(\succ_{i}, P_{i}(h)\right)$, then the greedy action of clinching the top payoff is obviously dominant at $h$. What remains to be shown is if $\operatorname{Top}\left(\succ_{i}, C_{i}(h)\right) \neq \operatorname{Top}\left(\succ_{i}, P_{i}(h)\right)$, then passing is obviously dominant at $h$.

Assume that there exists a history $h$ that is on the path of play for type $\succ_{i}$ when she follows the greedy strategy and $\operatorname{Top}\left(\succ_{i}, C_{i}(h)\right) \neq \operatorname{Top}\left(\succ_{i}, P_{i}(h)\right)$, yet passing is not obviously dominant at $h$; further, let $h$ be any earliest such history for which this is true. To shorten notation, let $x_{P}(h)=\operatorname{Top}\left(\succ_{i}, P_{i}(h)\right), x_{C}(h)=\operatorname{Top}\left(\succ_{i}, C_{i}(h)\right)$, and let $x_{W}(h)$ be the worst possible outcome from passing (and following the greedy strategy in the future). Since passing is not obviously dominant, it must be that $x_{W}(h) Z_{i} x_{C}(h)$.

First, note that $h^{\prime} \in \mathcal{H}_{i}(h)$ implies $x_{W}(h) \succsim_{i} x_{W}\left(h^{\prime}\right)$. Since passing is obviously dominant at all $h^{\prime} \in \mathcal{H}_{i}(h)$, we have $x_{W}\left(h^{\prime}\right) \succsim_{i} x_{C}\left(h^{\prime}\right)$, and together, these imply that $x_{W}(h) \succsim_{i} x_{C}\left(h^{\prime}\right)$ for all $h^{\prime} \in \mathcal{H}_{i}(h)$. At $h$, since passing is not obviously dominant, we have $x_{C}(h) \succ_{i} x_{W}(h)$, and further, there must be some $x^{\prime} \in P_{i}(h) \backslash G_{i}(h)$ such that $x^{\prime} \succ_{i} x_{C}(h) \succ_{i} x_{W}(h){ }^{40}$ The above implies that $x^{\prime} \succ_{i} x_{C}(h) \succ_{i} x_{C}\left(h^{\prime}\right)$ for all $h^{\prime} \in \mathcal{H}_{i}(h)$. Let $X_{0}=\left\{x^{\prime}: x^{\prime} \in\right.$ $P_{i}(h)$ and $\left.x^{\prime} \succ_{i} x_{C}(h)\right\}$. In words, $X_{0}$ is a set of payoffs that are possible at all $h^{\prime} \subseteq h$, and are strictly better than anything that was clinchable at any $h^{\prime} \subseteq h$ (and therefore have never been clinchable themselves). Order the elements in $X_{0}$ according to $\succ_{i}$, and wlog, let $x_{1} \succ_{i} x_{2} \succ_{i} \cdots \succ_{i} x_{M}$.

Definition. Let $h^{\prime}$ be a history where either agent $i$ is called to move or $h^{\prime}$ is a terminal history. Payoff $x$ becomes impossible for $i$ at $h^{\prime}$ if: (i) $x \in P_{i}\left(h^{\prime \prime}\right)$ for all $h^{\prime \prime} \in \mathcal{H}_{i}\left(h^{\prime}\right)$ and (ii) $x \notin P_{i}\left(h^{\prime}\right)$.

In other words, the history $h^{\prime}$ at which a payoff becomes impossible is the earliest history at which $i$ moves and where $x$ "disappears" as a possible outcome for her.

Consider a path of play starting from $h$ and ending in a terminal history $\bar{h}$ at which type $\succ_{i}$ of agent $i$ receives his worst case outcome $x_{W}(h)$. For every $x_{m} \in X_{0}$, let $h_{m}$ denote the history on this path at which $x_{m}$ becomes impossible for $i$. Note that because

\footnotetext{
${ }^{40}$ At least one such $x^{\prime}$ exists by the assumption that $\operatorname{Top}\left(\succ_{i}, C_{i}(h)\right) \neq \operatorname{Top}\left(\succ_{i}, P_{i}(h)\right)$, though there in general may be multiple such $x^{\prime}$.
} 
$i$ is ultimately receiving payoff $x_{W}(h)$, such a history $h_{m}$ exists for all $x_{m} \in X_{0} \cdot{ }^{41}$ Let $\hat{h}=$ $\max \left\{h_{1}, h_{2}, \ldots, h_{M}\right\}$ (ordered by $\subset$ ); in words, $\hat{h}$ is the earliest history at which everything in $X_{0}$ is no longer possible. Further, let $\hat{h}_{-m}=\max \left\{h_{1}, \ldots, h_{m-1}\right\}$, i.e., $\hat{h}_{-m}$ is the earliest history at which all payoffs strictly preferred to $x_{m}$ are no longer possible.

Claim 1. For all $x_{m} \in X_{0}$ and all $h^{\prime} \subseteq \bar{h}$, we have $x_{m} \notin C_{i}\left(h^{\prime}\right)$.

Proof. First, note that $x_{m} \notin C_{i}\left(h^{\prime}\right)$ for any $h^{\prime} \subseteq h$ by construction. We will show that $x_{m} \notin C_{i}\left(h^{\prime}\right)$ at any $\bar{h} \supseteq h^{\prime} \supset h$ as well. Start by considering $m=1$, and assume $x_{1} \in C_{i}\left(h^{\prime}\right)$ for some $\bar{h} \supseteq h^{\prime} \supset h$. By definition, $x_{1}=\operatorname{Top}\left(\succ_{i}, P_{i}(h)\right)$; since $h^{\prime} \supset h$ implies that $P_{i}\left(h^{\prime}\right) \subseteq$ $P_{i}(h)$, we have that $x_{1}=\operatorname{Top}\left(\succ_{i}, P_{i}\left(h^{\prime}\right)\right)$ as well. Since $x_{1} \in C_{i}\left(h^{\prime}\right)$ by supposition, greedy strategies direct $i$ to clinch $x_{1}$, which contradicts that she receives $x_{W}(h) .{ }^{42}$

Now, consider an arbitrary $m$, and assume that for all $m^{\prime}=1, \ldots, m-1$, payoff $x_{m^{\prime}}$ is not clinchable at any $h^{\prime} \subseteq \bar{h}$, but $x_{m}$ is clinchable at some $h^{\prime} \subseteq \bar{h}$. Let $x_{m^{\prime}}$ be (a) payoff that becomes impossible at $\hat{h}_{-m}$ and is such that $x_{m^{\prime}} \succ_{i} x_{m}$. There are two cases:

Case (i): $h^{\prime} \subset \hat{h}_{-m}$. This is the case where $x_{m}$ is clinchable while there is some strictly preferred payoff $x_{m^{\prime}} \succ_{i} x_{m}$ that is still possible. Note that at history $\hat{h}_{-m}$, neither (a) nor (b) in the definition of a millipede game hold for $x_{m^{\prime}}$. Further, by the inductive hypothesis, $x_{m^{\prime}}$ is nowhere clinchable, and so (c) does not hold either. This implies that (d) must hold, and so $x_{m}$ must be clinchable at $\hat{h}_{-m}$. Then, since all preferred payoffs are no longer possible at $\hat{h}_{-m}, x_{m}$ is the best possible payoff remaining, and is clinchable. Therefore, greedy strategies instruct agent $i$ to clinch $x_{m}$, which contradicts that she receives $x_{W}(h)$.

Case (ii): $h^{\prime} \supseteq \hat{h}_{-m}$. In this case, $x_{m}$ becomes clinchable after all strictly preferred payoffs are no longer possible. Thus, again, greedy strategies instruct $i$ to clinch $x_{m}$, which contradicts that she is receiving $x_{W}(h)$.

To finish the proof, again let $\hat{h}=\max \left\{h_{1}, h_{2}, \ldots, h_{M}\right\}$ and let $\hat{x}$ be a payoff that becomes impossible at $\hat{h}$. The claim shows that $\hat{x}$ is not clinchable at any $h^{\prime} \subseteq \hat{h}$. The preceding two statements imply that conditions (a)-(c) in the definition of a millipede game do not hold at $\hat{h}$, and so condition (d) must hold, i.e., $x_{C}(h) \in C_{i}(\hat{h})$. Since $x_{C}(h)$ is the best possible remaining payoff at $\hat{h}$, greedy strategies direct $i$ to clinch $x_{C}(h)$, which contradicts that she receives $x_{W}(h) .{ }^{43}$

\footnotetext{
${ }^{41}$ It is possible that $h_{m}$ is a terminal history.

${ }^{42}$ Recall that for terminal histories $h$, we define $C_{i}(h)=\{x\}$, where $x$ is the unique payoff associated with the terminal history. Thus, if $h^{\prime}$ is a terminal history, then $i$ receives payoff $x_{1}$, which also contradicts that she receives payoff $x_{W}(h)$.

${ }^{43}$ If $\hat{h}$ is a terminal history, then we make an argument analogous to footnote 42 to reach the same contradiction.
} 
We now prove the second part of the theorem, restated below as Proposition 2. To do so, we first need to introduce the pruning principle of Li (2017), which will simplify some of the arguments. Given a game $\Gamma$ and strategy profile $\left(S_{i}\left(\succ_{i}\right)\right)_{i \in \mathcal{N}}$, the pruning of $\Gamma$ with respect to $\left(S_{i}\left(\succ_{i}\right)\right)_{i \in \mathcal{N}}$ is a game $\Gamma^{\prime}$ that is defined by starting with $\Gamma$ and deleting all histories of $\Gamma$ that are never reached for any type profile. Then, the pruning principle says that if $\left(S_{i}\left(\succ_{i}\right)\right)_{i \in \mathcal{N}}$ is obviously dominant for $\Gamma$, the restriction of $\left(S_{i}\left(\succ_{i}\right)\right)_{i \in \mathcal{N}}$ to $\Gamma^{\prime}$ is obviously dominant for $\Gamma^{\prime}$, and both games result in the same outcome. Thus, for any OSP mechanism, we can find an equivalent OSP pruned mechanism. When proving this proposition, we assume that all OSP games have been pruned with respect to the equilibrium strategy profile. Note also that we actually prove a slightly stronger statement, which is that every OSP game is equivalent to a millipede game that satisfies the following additional property: for all $i$, all $h$ at which $i$ moves, and all $x \in G_{i}(h)$, there exists an action $a_{x} \in A(h)$ that clinches $x .^{44}$

Proposition 2. Every obviously strategy-proof mechanism $\left(\Gamma, S_{\mathcal{N}}\right)$ is equivalent to a millipede game with the greedy strategy.

Proof. The proof of this proposition is broken down into several lemmas.

Lemma 1. Every OSP game is equivalent to an OSP game with perfect information in which Nature moves at most once, as the first mover.

Proof. Ashlagi and Gonczarowski (2016) briefly mention this result in a footnote; here, we provide the straightforward proof for completeness. We first show that every OSP game is equivalent to an OSP game with perfect information. Denote by $A(\mathcal{I})$ the set of actions available at information set $\mathcal{I}$ to the agent who moves at $\mathcal{I}$. Take an obviously strategy-proof game $\Gamma$ and consider its perfect-information counterpart $\Gamma^{\prime}$, that is the perfect information game at which at every history $h$ in $\Gamma$ the moving agent's information set is $\{h\}$ in $\Gamma^{\prime}$, the available actions are $A(\mathcal{I})$, and the outcomes in $\Gamma^{\prime}$ following any terminal history are the same as in $\Gamma$. Notice that the support of possible outcomes at any history $h$ in $\Gamma^{\prime}$ is a subset of the support of possible outcomes at $\mathcal{I}(h)$ in $\Gamma$. Thus, the worst-case outcome from any action (weakly) increases in $\Gamma^{\prime}$, while the best-case outcome (weakly) decreases. Thus, if there is an obviously dominant strategy in $\Gamma$, following the analogous strategy in $\Gamma^{\prime}$ continues to be obviously dominant. Hence, $\Gamma^{\prime}$ is obviously strategy-proof and equivalent to $\Gamma$.

We now show that every OSP game is equivalent to a perfect-information OSP game in which Nature moves once, as the first mover. Consider a game $\Gamma$, which, by the previous paragraph, we can assume has perfect information. Let $\mathcal{H}_{\text {nature }}$ be the set of histories $h$ at which Nature moves in $\Gamma$. Consider a modified game $\Gamma^{\prime}$ in which at the empty history Nature

\footnotetext{
${ }^{44}$ See Lemma 3 below.
} 
chooses actions from $\times_{h \in \mathcal{H}_{\text {nature }}} A(h)$. After each of Nature's initial moves, we replicate the original game, except at each history $h$ at which Nature is called to play, we delete Nature's move and continue with the subgame corresponding to the action Nature chose from $A(h)$ at $\emptyset$. Again, note that for any agent $i$ and history $h$ at which $i$ is called to act, the support of possible outcomes at $h$ in $\Gamma^{\prime}$ is a subset of the support of possible outcomes at the corresponding history in $\Gamma$ (where the corresponding histories are defined by mapping the $A(h)$ component of the action taken at $\emptyset$ by Nature in $\Gamma^{\prime}$ as an action made by Nature at $h$ in game $\Gamma$ ). Using reasoning similar to the previous paragraph, we conclude that $\Gamma^{\prime}$ is obviously strategy-proof, and $\Gamma$ and $\Gamma^{\prime}$ are equivalent.

Lemma 2. Let $\Gamma$ be an obviously strategy-proof game of perfect information that is pruned with respect to the obviously dominant strategy profile $\left(S_{i}\left(\succ_{i}\right)\right)_{i \in \mathcal{N}}$. At any history $h$ where an agent $i$ is called to move, there is at most one action $a^{*} \in A(h)$ such that $P_{i}\left(\left(h, a^{*}\right)\right) \nsubseteq G_{i}(h)$.

Proof. We prove this result via two steps.

Step 1. Suppose there are two distinct payoffs $x, y \in P_{i}(h) \backslash G_{i}(h)$ and a preference type $\succ_{i}$ such that (i) $x$ and $y$ are the first and second $\succ_{i}$-best possible payoffs in $P_{i}(h)$, and (ii) $h$ is on the path of the game for type $\succ_{i}$. Then, there is at most one action $a^{*} \in A(h)$ such that $P_{i}\left(\left(h, a^{*}\right)\right) \nsubseteq G_{i}(h)$, and type $\succ_{i}$ must choose action $a^{*}$ at $h$.

To prove the claim of this step, it is enough to consider the case $x \succ_{i} y$. First, note that if $x \in P_{i}((h, a))$ for some $a \in A(h)$, then $y \in P_{i}((h, a))$ as well. Indeed, if not then $x \in P_{i}((h, a))$ and $y \notin P_{i}((h, a))$. For type $\succ_{i}$, the worst case outcome from following $a$ is strictly worse than $y$ because $x$ and $y$ are assumed to be the $\succ_{i}$-best possible payoffs in $P_{i}(h), x$ is not guaranteeable at $h$, and $y$ is not possible following $a$. Because $y \in P_{i}(h)$, action $a$ is not obviously dominant. As $x$ is not guaranteeable at $h$, the worst case outcome from any other $a^{\prime} \neq a$ is strictly worse than $x$, while the best case outcome from $a$ is $x$, and so no $a^{\prime}$ can obviously dominate $a$. Thus, type $\succ_{i}$ has no obviously dominant action, which contradicts that the game is OSP, and proves the claim of this paragraph.

Now, assume that there are two actions $a_{1}^{*}$ and $a_{2}^{*}$ such that $P_{i}\left(\left(h, a_{j}^{*}\right)\right) \nsubseteq G_{i}(h)$ for $j=1,2$. Consider some $x \in P_{i}(h) \backslash G_{i}(h)$. By the previous paragraph, we know that $x \in P_{i}\left(\left(h, a_{1}^{*}\right)\right)$ and $x \in P_{i}\left(\left(h, a_{2}^{*}\right)\right)$. However, by assumption, $x$ is not guaranteeable at $h$, and so, for type $\succ_{i}$, the worst case payoff from any action $a^{\prime}$ must be strictly worse than $x$, while the best case outcomes from $a_{1}^{*}$ and $a_{2}^{*}$ are both $x$. Therefore, no action $a^{\prime} \in A(h)$ is obviously dominant, which contradicts that $\Gamma$ is OSP. ${ }^{45}$

\footnotetext{
${ }^{45}$ Note that if there were only one such action $a_{1}^{*}$, then it would still be true that there is no action that obviously dominates $a_{1}^{*}$ for this type. However, $a_{1}^{*}$ itself might be obviously dominant. When there are two such actions, $a_{1}^{*}$ does not obviously dominate $a_{2}^{*}$, nor does $a_{2}^{*}$ obviously dominate $a_{1}^{*}$, and thus there are no obviously dominant actions.
} 
We can conclude that there is at most one action $a^{*}$ that leads to $x$ for some continuation strategies of players. Because $x$ is only possible following $a^{*}$, if an obviously dominant strategy profile exists, any type $\succ_{i}$ that ranks any $x \in P_{i}(h) \backslash G_{i}(h)$ first among the payoffs in $P_{i}(h)$ must select $a^{*}$ at this history, concluding the proof of the claim of Step 1.

Step 2. Suppose $i$ moves at history $h$. Then, there is at most one action $a^{*} \in A(h)$ such that $P_{i}\left(\left(h, a^{*}\right)\right) \nsubseteq G_{i}(h)$.

To prove this step, first consider any earliest history $h_{0}^{i}$ at which $i$ is to move. Note that since this is an earliest history for $i$, history $h_{0}^{i}$ is on the path of play for all types of agent $i$. If $P_{i}\left(h_{0}^{i}\right) \backslash G_{i}\left(h_{0}^{i}\right)=\{x\}$ and there were two actions $a_{1}^{*}$ and $a_{2}^{*}$ as in the statement, then for any type that ranks $x$ first, the worst case from any action is strictly worse than $x$ (because $x$ is not guaranteeable), while the best case from both $a_{1}^{*}$ and $a_{2}^{*}$ is $x$, so nothing can obviously dominate $a_{1}^{*}$; by similar reasoning, $a_{1}^{*}$ does not obviously dominate $a_{2}^{*}$. If there are two payoffs $x, y \in P_{i}\left(h_{0}^{i}\right) \backslash G_{i}\left(h_{0}^{i}\right)$, then we can apply Step 1 to type $x \succ_{i} y \succ_{i} \cdots$.

Now, consider any successor history $h^{\prime} \supset h_{0}^{i}$ at which $i$ is to move, and make the inductive assumption that at every $h \subset h^{\prime}$, there is only one possible action $a^{*} \in A(h)$ such that $P_{i}\left(\left(h, a^{*}\right)\right) \nsubseteq G_{i}(h)$. First, consider the case where $\left|P_{i}\left(h^{\prime}\right) \backslash G_{i}\left(h^{\prime}\right)\right|=1$, and let $x$ be the unique payoff that is possible but not guaranteeable. By way of contradiction, assume there were two actions, $a_{1}^{*}$ and $a_{2}^{*}$, such that $x$ was a possible outcome. By the pruning principle, some type $\succ_{i}$ must be receiving $x$ at some terminal history $\bar{h} \supset h^{\prime}$. If $x$ is the top choice among all payoffs in $P_{i}\left(h^{\prime}\right)$ for some type $\succ_{i}$ for which history $h^{\prime}$ is on-path, then, following similar reasoning as above, neither $a_{1}^{*}$ nor $a_{2}^{*}$ can be obviously dominant. If $x$ is not the best possible payoff in $P_{i}\left(h^{\prime}\right)$ for any such type, then, since $x$ is the only payoff that is possible, but not guaranteeable, every other payoff in $P_{i}\left(h^{\prime}\right)$ is guaranteeable. This implies that every type of agent $i$ for which $h^{\prime}$ is on-path can guarantee herself her best possible outcome in $P_{i}\left(h^{\prime}\right)$, and so no type should ever play a strategy for which she ends up receiving a payoff of $x$ at any terminal history $\bar{h} \supset h^{\prime}$, which is a contradiction.

Finally, assume $\left|P_{i}\left(h^{\prime}\right) \backslash G_{i}\left(h^{\prime}\right)\right| \geq 2$, and let $x, y \in P_{i}\left(h^{\prime}\right) \backslash G_{i}\left(h^{\prime}\right)$. First, consider the case that there is some $x \in P_{i}\left(h^{\prime}\right) \backslash G_{i}\left(h^{\prime}\right)$ such that $x \notin G_{i}(h)$ for all $h \subset h^{\prime}$ at which $i$ is to move. Recall the inductive hypothesis says that at all such $h$, there is a unique action such that $P_{i}\left(\left(h, a^{*}\right)\right) \nsubseteq G_{i}(h)$. Thus, if $x$ has never been guaranteeable, all types $x \succ_{i} \cdots$ must have followed this unique action at all such $h \subset h^{\prime}$, and we can apply Step 1 to the type $x \succ_{i} y \succ_{i} \cdots$. The last case to consider is where all $x, y \in P_{i}\left(h^{\prime}\right) \backslash G_{i}\left(h^{\prime}\right)$ were also guaranteeable at some earlier history $h$. Consider a type $\succ_{i}$ of agent $i$ who receives payoff $x$ at some terminal history $\bar{h} \supset h^{\prime}$. First, note that for any $z \succ_{i} x, z \notin G_{i}\left(h^{\prime}\right)$ as otherwise this type would not follow a strategy whereby $x$ was a possible outcome. ${ }^{46}$ Let $z$ be the $\succ_{i}$-best

\footnotetext{
${ }^{46}$ If $x$ is the $\succ_{i}$-best possible payoff in $P_{i}\left(h^{\prime}\right)$ for all types that reach $h^{\prime}$, then apply the same argument to
} 
payoff in $P_{i}\left(h^{\prime}\right)$, and $w$ be the second-best possible payoff in $P_{i}\left(h^{\prime}\right)$ for this type; we allow $w=x$. We can again apply Step 1 to type $\succ_{i}$ and conclude there is at most one action $a^{*}$ such that $P_{i}\left(\left(h, a^{*}\right)\right) \nsubseteq G_{i}(h)$.

Clinching actions are those for which $i$ 's payoff is completely determined after following the action. Lemma 2 shows that if a game is OSP, then at every history, for all actions $a$ with the exception of possibly one special action $a^{*}$, all payoffs that are possible following $a$ are also guaranteeable at $h$; note, however, it does not say that all actions but at most one are clinching actions. Indeed, it leaves open the possibility that there are several actions that can ultimately lead to multiple final payoffs for $i$, which can happen when different payoffs are guaranteeable for $i$ by following different actions in the future of the game. The next lemma shows that if this is the case, we can always construct an equivalent OSP game such that all actions except for possibly one are clinching actions.

Lemma 3. Let $\Gamma$ be an obviously strategy-proof game of perfect information that is pruned with respect to the strategy profile $\left(S_{i}\left(\succ_{i}\right)\right)_{i \in \mathcal{N}}$. There exists an equivalent obviously strategyproof game $\Gamma^{\prime}$ with perfect information such that:

(i) At each history $h$, at least $|A(h)|-1$ actions at $h$ are clinching actions.

(ii) For every payoff $x \in G_{i}(h)$, there exists an action $a_{x} \in A(h)$ that clinches $x$.

Proof. Consider some history $h$ of game $\Gamma$ at which $i$ moves. By Lemma 2, all but at most one action (denoted $a^{*}$ ) in $A(h)$ satisfy $P_{i}((h, a)) \subseteq G_{i}(h)$; this means that any obviously dominant strategy for type $\succ_{i}$ that does not choose $a^{*}$ guarantees the best possible outcome in $P_{i}(h)$ for type $\succ_{i}$. Thus the set $\mathcal{S}_{i}(h)=\left\{S_{i}:\left|X\left(h, S_{i}\right)\right|=1\right\}$ contains all possible obviously dominant strategies of agent $i$ for which $h$ is on path and that do not choose $a^{*}$. Notice that $\mathcal{S}_{i}(h)$ is the set of strategies that guarantee some payoff $x$ for $i$ if $i$ plays strategy $S_{i}$ starting from history $h$.

We create a new game $\Gamma^{\prime}$ that is the same as $\Gamma$, except we replace the subgame starting from history $h$ with a new subgame defined as follows. If there is an action $a^{*}$ such that $P_{i}\left(\left(h, a^{*}\right)\right) \nsubseteq G_{i}(h)$ in the original game (of which there can be at most one), then there is an analogous action $a^{*}$ in the new game, and the subgame following $a^{*}$ is exactly the same as in the original game $\Gamma$. Additionally, there are $M=\left|\mathcal{S}_{i}(h)\right|$ other actions at $h$, denoted $a_{1}, \ldots, a_{M}$. Each $a_{m}$ corresponds to one strategy $S_{i}^{m} \in \mathcal{S}_{i}(h)$, and following each $a_{m}$, we replicate the original game, except that at any future history $h^{\prime} \supseteq h$ at which $i$ is called on to act, all actions (and their subgames) are deleted and replaced with the subgame starting with the action $a^{\prime}=S_{i}^{m}\left(h^{\prime}\right)$ that $i$ would have played in the original game had she followed a type that receives $y$ at some terminal history and set $z=x$. 
strategy $S_{i}^{m}(\cdot)$. In other words, if $i$ were to choose some action $a \neq a^{*}$ at $h$ in the original game, then, in the new game $\Gamma^{\prime}$, we ask agent $i$ to choose not only her current action, but all future actions that she would have chosen according to $S_{i}^{m}(\cdot)$ as well. By doing so, we have created a new game in which every action (except for $a^{*}$, if it exists) at $h$ clinches some payoff $x$, and further, agent $i$ is never called upon to move again. ${ }^{47}$

We construct strategies in $\Gamma^{\prime}$ that are the counterparts of strategies from $\Gamma$, so that for all agents $j \neq i$, they continue to follow the same action at every history as they did in the original game, and for $i$, at history $h$ in the new game, she takes the action $a_{m}$ that is associated with the strategy $S_{i}^{m}$ in the original game. By definition if all the agents follow strategies in the new game analogous to the their strategies from the original game, the same terminal history will be reached, and so $\Gamma$ and $\Gamma^{\prime}$ are equivalent under their respective strategy profiles.

We must also show that if a strategy profile is obviously dominant for $\Gamma$, this modified strategy profile is obviously dominant for $\Gamma^{\prime}$. To see why the modified strategy profile is obviously dominant for $i$, note that if her obviously dominant action in the original game was part of a strategy that guarantees some payoff $x$, she now is able to clinch $x$ immediately, which is clearly obviously dominant; if her obviously dominant strategy was to follow a strategy that did not guarantee some payoff $x$ at $h$, this strategy must have directed $i$ to follow $a^{*}$ at $h$. However, in $\Gamma^{\prime}$, the subgame following $a^{*}$ is unchanged relative to $\Gamma$, and so $i$ is able to perfectly replicate this strategy, which obviously dominates following any of the clinching actions at $h$ in $\Gamma^{\prime}$. In addition, the game is also obviously strategy-proof for all $j \neq i$ because, prior to $h$, the set of possible payoffs for $j$ is unchanged, while for any history succeeding $h$ where $j$ is to move, having $i$ make all of her choices earlier in the game only shrinks the set of possible outcomes for $j$, in the set inclusion sense. When the set of possible outcomes shrinks, the best possible payoff from any given strategy only decreases (according to $j$ 's preferences) and the worst possible payoff only increases, and so, if a strategy was obviously dominant in the original game, it will continue to be so in the new game. Repeating this process for every history $h$, we are left with a new game where, at each history, there are only clinching actions plus (possibly) one passing action, and further, every payoff that is guaranteeable at $h$ is also clinchable at $h$.

Lemma 4. Let $\Gamma$ be an obviously strategy-proof game that is pruned with respect to the obviously dominant strategy profile $S_{\mathcal{N}}$ and that satisfies Lemmas 1 and 3. At every history $h^{i}$

\footnotetext{
${ }^{47}$ More precisely, all of $i$ 's future moves are trivial moves in which she has only one possible action; hence these histories may further be removed to create an equivalent game in which $i$ is never called on to move again. Note that this only applies to the actions $a \neq a^{*}$; it is still possible for $i$ to follow $a^{*}$ at $h$ and be called upon to make a non-trivial move again later in the game.
} 
at which an agent $i$ moves and every terminal history $\bar{h}$, for every payoff $z$, one of conditions (a)-(d) must hold.

Proof. Assume not. First, consider the case of a non-terminal history $h^{i}$ where $i$ is called to move and a payoff $z$ be such that (a), (b) and (c) do not hold at $h^{i}$, i.e., the following are true:

$$
\begin{aligned}
& \text { (a') } z \notin P_{i}\left(h^{i}\right) \\
& \text { (b') } z \in P_{i}(h) \text { for all } h \in \mathcal{H}_{i}\left(h^{i}\right) \\
& \text { (c') } z \notin \cup_{\tilde{h} \in \mathcal{H}_{i}\left(h^{i}\right)} C_{i}(\tilde{h}) \text { and }
\end{aligned}
$$

Points (b') and (c') imply that $z$ is possible at every $h \subsetneq h^{i}$ where $i$ is to move, but it is not clinchable at any of them. This implies that for any type of agent $i$ that ranks $z$ first, any obviously dominant strategy must have the agent passing at all $h \in \mathcal{H}_{i}\left(h^{i}\right) .{ }^{48}$

Towards a contradiction, assume that (d) did not hold, i.e., there exists some $h^{\prime} \in \mathcal{H}_{i}\left(h^{i}\right)$ and $x \in C_{i}\left(h^{\prime}\right)$ such that $x \notin C_{i}\left(h^{i}\right)$. Consider a type $z \succ_{i} x \succ_{i} \cdots$. We argue that if $(\mathrm{d})$ does not hold at $h^{i}$, then there is some $\hat{h}^{i} \subsetneq h^{i}$ such that type $\succ_{i}$ has no obviously dominant action. First, note that at any such $\hat{h}^{i} \subsetneq h^{i}$, no clinching action can be obviously dominant, because $z$ is always possible following the passing action, but is never clinchable, and so the worst case from clinching is strictly worse than the best case from passing, which is $z$. Next, there must be some $\hat{h}^{i} \subsetneq h^{i}$ such that the passing action also is not obviously dominant. To see why, note that $h^{i}$ must be on the path of play for type $\succ_{i}$, since she must pass at all $h^{\prime} \subsetneq h^{i}$. By assumption, $z \notin P_{i}\left(h^{i}\right)$ and $x \notin C_{i}\left(h^{i}\right)$, which implies that the worst case outcome from passing at any $h^{\prime} \subsetneq h^{i}$ is some $y$ that is strictly worse than $x$ according to $\succ_{i}$. However, we also have $x \in C_{i}\left(\hat{h}^{i}\right)$ for some $\hat{h}^{i} \subsetneq h^{i}$, and so, the best case outcome from clinching $x$ at $\hat{h}^{i}$ is $x$. This implies that passing is not obviously dominant, which contradicts that $\Gamma$ is OSP.

Last, consider a terminal history $\bar{h}$. As above, let $z$ be a payoff such that (a'), (b'), and (c') hold (i.e., (a), (b), and (c) are false). By definition, $C_{i}(\bar{h})=\{y\}$ for all $i$, where $y$ is the unique outcome associated with terminal history $\bar{h}$ (note also that $z \notin P_{i}(\bar{h})$ implies that $y \neq z)$. Assume that $(\mathrm{d})$ does not hold, i.e., there exists some $h^{\prime} \in \mathcal{H}_{i}(\bar{h})$ and $x \in C_{i}\left(h^{\prime}\right)$ such that $x \notin C_{i}(\bar{h})$. Note that (i) $z \neq y$ (because $z \notin P_{i}(\bar{h})$ ); (ii) $z \neq x$ (by $\left(\mathrm{c}^{\prime}\right)$ ); and (iii) $x \neq y$ (because $x \notin C_{i}(\bar{h})$ ). In other words, $x, y, z$ must all be distinct payoffs. Consider the type $z \succ_{i} x \succ_{i} y \succ_{i} \cdots$. By (b') and (c'), $z$ is possible at every $h \subsetneq \bar{h}$ where $i$ is to move, but is not clinchable at any such history. Thus, any obviously dominant strategy of type $\succ_{i}$

\footnotetext{
${ }^{48}$ At all such $h$, since $z$ is not clinchable, but is possible, it must be possible following the (unique) passing action. This means that best case outcome from passing is $z$, while the worst case outcome from clinching is strictly worse than $z$. Thus, no clinching action can be obviously dominant, so, if an obviously dominant strategy exists, it must instruct $i$ to pass.
} 
must have agent $i$ passing at any such history. However, at $h^{\prime}, i$ could have clinched $x$, and so passing is not obviously dominant (because $y$ is possible from passing).

Proposition 2 follows from Lemmas 1, 3, and 4. Theorem 1 then follows from Propositions 1 and 2.

\section{A.2 Proof of Theorem 2}

Consider any two strategies $S_{i}$ and $S_{i}^{\prime}$ of agent $i$; let $h$ be the earliest point of departure for these two strategies.

Suppose $S_{i}$ is strongly obviously strategy-proof in $\Gamma$. Then any outcome that is possible after playing $S_{i}$ is weakly better than any outcome that is possible after playing $S_{i}^{\prime}$ in game $\Gamma^{\prime}$, and hence in the outcome set-equivalent game $\Gamma^{\prime}$. Hence, $S_{i}$ strongly obviously dominates $S_{i}^{\prime}$ in game $\Gamma^{\prime}$, and thus it weakly dominates it.

Now suppose $S_{i}$ weakly dominates $S_{i}^{\prime}$ in all games $\Gamma^{\prime}$ that are outcome equivalent to $\Gamma$. Consider such a $\Gamma^{\prime}$ in which all moves of agent $i$ following history $h$ are made by Nature instead. Since, $S_{i}$ weakly dominates $S_{i}^{\prime}$ in $\Gamma^{\prime}$, we conclude that any outcome that is possible after playing $S_{i}$ is weakly better than any outcome that is possible after playing $S_{i}^{\prime}$ in game $\Gamma^{\prime}$, and hence in the outcome set-equivalent game $\Gamma$. Hence, $S_{i}$ strongly obviously dominates $S_{i}^{\prime}$ in game $\Gamma$.

\section{A.3 Proof of Theorem 3}

We first note the following lemma, which says that the first time an agent is called to play (at a nontrivial history) in an SOSP game, all of her available actions are associated with a unique possible payoff, except for possibly one action, which may have two possible payoffs.

Lemma 5. Let $\Gamma$ be a pruned SOSP game. Let $h_{0}^{i}$ be any earliest history at which agent $i$ is called to play and has at least two actions, i.e., $\left|A\left(h_{0}^{i}\right)\right| \geq 2$. Then, $\left|P_{i}\left(\left(h_{0}^{i}, a\right)\right)\right| \leq 2$ for all $a \in A\left(h_{0}^{i}\right)$, with equality for at most one $a \in A\left(h_{0}^{i}\right)$.

Proof of lemma. Since $h_{0}^{i}$ is the first time $i$ is called to move, it is on-path for all types of agent $i$. We first show that $\left|P_{i}\left(\left(h_{0}^{i}, a\right)\right)\right| \leq 2$ for all $a \in A\left(h_{0}^{i}\right)$. Assume not, which means that there exists some $a \in A(h)$ such that $\left|P_{i}\left(\left(h_{0}^{i}, a\right)\right)\right| \geq 3$. Let $x, y, z \in P_{i}\left(\left(h_{0}^{i}, a\right)\right)$ be three distinct payoffs that are possible following $a$. There must be some payoff in $P_{i}\left(\left(h_{0}^{i}, a\right)\right)$, say $x$, 
such that for all $a^{\prime} \in A\left(h_{0}^{i}\right), P_{i}\left(\left(h_{0}^{i}, a^{\prime}\right)\right) \neq\{x\} .{ }^{49}$ Consider some action $a^{\prime} \neq a$. If there exists some $w \in P_{i}\left(\left(h_{0}^{i}, a^{\prime}\right)\right)$ such that $w \neq x, y$, consider the type $x \succ_{i} w \succ_{i} y \succ_{i} \cdots$, and note that this type has no strongly obviously dominant action. ${ }^{50}$ Otherwise, $P_{i}\left(\left(h_{0}^{i}, a^{\prime}\right)\right)=\{x, y\}$ or $\{y\}$; in either case, type $x \succ_{i} y \succ_{i} z \succ_{i} \cdots$ has no strongly obviously dominant action. ${ }^{51}$

Finally, we show that $\left|P_{i}\left(\left(h_{0}^{i}, a\right)\right)\right|=2$ for at most one $a \in A\left(h_{0}^{i}\right)$. Let $a$ and $a^{\prime}$ be two actions such that there are two possible payoffs for $i$ following each, and take two distinct payoffs $x, y$ such that $x \in P_{i}\left(h_{0}^{i}, a\right)$ and $y \in P_{i}\left(h_{0}^{i}, a^{\prime}\right)$. There are two cases.

Case (i): There exists some $z \neq x, y$ such that $z \in P_{i}\left(\left(h_{0}^{i}, a\right)\right) \cup P_{i}\left(\left(h_{0}^{i}, a^{\prime}\right)\right)$. Without loss of generality, assume that $z \in P_{i}\left(\left(h_{0}^{i}, a\right)\right)$, which, by the first part of the theorem, means $P_{i}\left(\left(h_{0}^{i}, a\right)\right)=\{x, z\}$. Similar to the above, we can assume that $P_{i}\left(\left(h_{0}^{i}, a^{\prime \prime}\right)\right) \neq\{x\}$ for all $a^{\prime \prime} \in A\left(h_{0}^{i}\right)$ (by pruning). Thus, type $x \succ_{i} y \succ_{i} z \succ_{i} \cdots$ has no strongly obviously dominant action. $^{52}$

Case (ii): There does not exist any $z$ as defined in case $(i)$. In this case, $P_{i}\left(\left(h_{0}^{i}, a\right)\right)=$ $P_{i}\left(\left(h_{0}^{i}, a^{\prime}\right)\right)=\{x, y\}$. Again, pruning implies that (without loss of generality) $P_{i}\left(\left(h_{0}^{i}, a^{\prime \prime}\right)\right) \neq$ $\{x\}$ for all $a^{\prime \prime} \in A\left(h_{0}^{i}\right)$. Then, type $x \succ_{i} y \succ_{i} \cdots$ has no strongly obviously dominant action. $^{53}$

Continuing with the main proof, if a history $h$ is payoff-relevant, then by definition $|A(h)| \geq 2$ and $\left|P_{i}(h)\right| \geq 2$. Assume that there was a path of the game with two payoffrelevant histories $h_{1} \subset h_{2}$ for some agent $i$, and note that it is without loss of generality to assume that $h_{1}$ and $h_{2}$ are the first and second times $i$ is called to play on the path, and that $\left|P_{i}\left(\left(h_{1}, a\right)\right)\right|>1$ for some $a \in A\left(h_{1}\right) .{ }^{54}$ By the lemma, there can only be one such action, denoted $a_{1}^{*}$, and $\left|P_{i}\left(\left(h_{1}, a_{1}^{*}\right)\right)\right|=2$. For notational purposes, define $P_{i}\left(h_{1}, a_{1}^{*}\right)=\{x, y\}$. By pruning, the following must hold for at least one of $w=x, y$ : there is no action $a \in A\left(h_{1}\right)$ such that $P_{i}\left(\left(h_{1}, a\right)\right)=\{w\} .{ }^{55}$ Without loss of generality, assume that this holds for $y$, and

\footnotetext{
${ }^{49}$ If there were such an $a^{\prime}$ for each payoff in $P_{i}\left(\left(h_{0}^{i}, a\right)\right)$, it would not be strongly obviously dominant for any type of agent $i$ to follow action $a$, and it can be pruned.

${ }^{50}$ Action $a$ does not strongly obviously dominate $a^{\prime}$, since $y$ is possible from $a$, whereas the best case from $a^{\prime}$ is at least $w$. Similarly, no other $a^{\prime \prime} \neq a$ can strongly obviously dominate $a$, since the worst case from any other action is strictly worse than $x$, while the best case from $a$ is $x$.

${ }^{51}$ The worst case from $a$ is at best $z$, while the best case from $a^{\prime}$ is at least $y$, and so $a$ does not strongly obviously dominate $a^{\prime}$. Similarly, the worst case from any $a^{\prime \prime} \neq a$ is at best $y$, and the best case from $a$ is $x$, and so no other $a^{\prime \prime}$ can strongly obviously dominate $a$.

${ }^{52}$ The worst case from $a$ is $z$, while $y$ is possible from $a^{\prime}$, and so $a$ is not strongly obviously dominant. The worst case from any other $a^{\prime \prime} \neq a$ is strictly worse than $x$, while the best case from $a$ is $x$, so no other $a^{\prime \prime}$ is strongly obviously dominant either.

${ }^{53}$ Neither $a$ nor $a^{\prime}$ strongly obviously dominate the other, since the worst case from either is $y$, while the best case from the other is $x$. Similarly, no other $a^{\prime \prime}$ strongly obviously dominates $a$, since the worst case from any such $a^{\prime \prime}$ is strictly worse than $x$, while the best case from $a$ is $x$.

${ }^{54}$ If $\left|P_{i}\left(\left(h_{1}, a\right)\right)\right|=1$ for all $a \in A\left(h_{1}\right)$ the first time $i$ is called to play, then it is clear that $\left|P_{i}\left(h^{\prime}\right)\right|=1$ for any $h^{\prime} \supset h_{1}$, and so $i$ has only one payoff-relevant history along any path that includes $h_{1}$.

${ }^{55}$ If there were such an action for both $x$ and $y$, then it would not be strongly obviously dominant for any
} 
note that this implies that no action $a_{1}^{\prime} \neq a_{1}^{*}$ can be strongly obviously dominant for any type that ranks $y$ first. In particular, if there exists some $z \neq x, y$ such that $z \in P_{i}\left(h_{1}\right)$, then type $y \succ_{i} z \succ_{i} \cdots$ does not have a strongly obviously dominant action at $h_{1}{ }^{56}$ Therefore, at $h_{1}$, we must have $P_{i}\left(\left(h_{1}, a_{1}^{*}\right)\right)=\{x, y\}$ and $P_{i}\left(\left(h_{1}, a_{1}^{\prime}\right)\right)=\{x\}$ for all $a_{1}^{\prime} \neq a_{1}^{*}$.

If $i$ follows $a_{1}^{\prime} \neq a_{1}^{*}$, then any potential future histories are payoff-irrelevant for $i$, and so $h_{2} \supseteq\left(h_{1}, a_{1}^{*}\right)$. Strong obvious dominance requires that every type $\succ_{i}$ that prefers $x$ to $y$ must select some action $a_{1}^{\prime} \neq a_{1}^{*}$ at $h_{1}$, and so history $h_{2}$ is only on the path of play for those types that prefer $y$ to $x$. Since $h_{2}$ is payoff-relevant, it must be that $P_{i}\left(h_{2}\right)=\{x, y\}$. Further, there can be no action $a_{2} \in A\left(h_{2}\right)$ such that $P_{i}\left(\left(h_{2}, a_{2}\right)\right)=\{y\}$; otherwise, all types who prefer $y$ to $x$ would have to follow such an action, and so any action for which $x$ is a possible outcome could be pruned, which contradicts $x \in P_{i}\left(h_{2}\right)$. Thus, for all actions $a \in A\left(h_{2}\right)$, either $P_{i}\left(\left(h_{2}, a\right)\right)=\{x, y\}$ or $\{x\}$. Pruning once again rules out the latter possibility, and so $P_{i}\left(\left(h_{2}, a\right)\right)=\{x, y\}$ for all $a \in A\left(h_{2}\right)$. If there are two such actions, then neither strongly obviously dominates the other; hence, $\left|A\left(h_{2}\right)\right|=1$, which contradicts that $h_{2}$ is payoff-relevant.

\section{A.4 Proof of Theorem 4}

That curated dictatorships are SOSP is immediate from the definition, and so we focus on proving that every SOSP game is equivalent to a curated dictatorship. Note first that the pruning principle (see the proof of Theorem 1) continues to apply to strong obvious dominance. Similarly, deleting trivial histories at which there is at most one action also preserves strong obvious dominance. Finally, following the same reasoning as in the proof of Theorem 1, given any SOSP game, we can construct an equivalent SOSP game of perfect information in which Nature moves at most once, as the first mover, and so we can focus on the deterministic subgame after any potential move by Nature. Thus, what remains to show is that every perfect-information, pruned SOSP game in which there are no moves by Nature and no trivial histories is equivalent to a curated dictatorship.

Let $\Gamma$ be such a game. By Theorem 3, each agent $i$ can have at most one payoff-relevant history along any path of game $\Gamma$, and this history (if it exists) is the first time $i$ is called to play. Consider any such history $h_{0}^{i}$. If there is some other history $h^{\prime} \supset h_{0}^{i}$ at which $i$ is called to play, then history $h^{\prime}$ must be payoff-irrelevant for $i$; in other words, there is some payoff $x$ such that $P_{i}\left(\left(h^{\prime}, a^{\prime}\right)\right)=\{x\}$ for all $a^{\prime} \in A\left(h^{\prime}\right)$. Using the same technique as in

type to follow action $a_{1}^{*}$, and it can be pruned.

${ }^{56}$ Note that $h_{1}$ is on-path for all types of agent $i$, since it is the first time $i$ is called to move. Action $a_{1}^{*}$ is not strongly obviously dominant, since the worst-case outcome is $x$, while by construction, there exists some other action $a_{1}^{\prime} \neq a_{1}^{*}$ where $z$ is possible. No action $a_{1}^{\prime} \neq a_{1}^{*}$ is strongly obviously dominant either, as just argued. 
the proof of Theorem 1, we can construct an equivalent game $\Gamma^{\prime}$ in which at history $h_{0}^{i}, i$ is asked to also choose her actions for all successor histories $h^{\prime} \supset h_{0}^{i}$ at which she might be called to play, and then is not called to play again after $h_{0}^{i}$ (see the proof of Theorem 1 for a more formal description of this procedure). Since all of these future histories were payoff-irrelevant for $i$, the new game continues to be strongly obvious dominant for $i$. Strong obvious dominance is also preserved for all $j \neq i$, since having $i$ make all of her choices earlier only shrinks the set of possible outcomes any time $j$ is called to move, and thus, if some action was strongly obviously dominant in the old game, the analogous action(s) will be strongly obviously dominant in the new game. Repeating this for every agent and every history, we have constructed a SOSP game $\Gamma^{\prime}$ that is equivalent to $\Gamma$ and in which each agent is called to move at most once along any path of play.

We claim that $\Gamma^{\prime}$ is a curated dictatorship. Assume not, and let $h$ be a history where the definition of a curated dictatorship is violated. First assume that $\left|P_{i}(h)\right| \geq 3$. Since $\Gamma^{\prime}$ is not a curated dictatorship, there must be some payoff $x \in P_{i}(h)$ that $i$ cannot clinch at $h$. This means that for any action $a \in A(h)$ such that $x \in P_{i}((h, a))$, there is some other $y \in P_{i}((h, a))$. Combining this with Lemma 5 (see the proof of Theorem 3) implies that at $h$, there is exactly one action $a \in A(h)$ such that $P_{i}((h, a))=\{x, y\}$, and, for all other $a^{\prime} \neq a, x \notin P_{i}\left(\left(h, a^{\prime}\right)\right)$. Let $z \neq x, y$ be some third payoff possible at $h$, and consider the type $x \succ_{i} z \succ_{i} y \succ_{i} \ldots .{ }^{57}$ For this type, $a$ is not strongly obviously dominant, since the worst-case from $a$ is $y$, while there is some other action for which $z$ is possible. Similarly, no other action $a^{\prime} \neq a$ is strongly obviously dominant either, since $x$ is not possible from any such $a^{\prime}$, but is possible from $a$. Thus, this type has no strongly obviously dominant action, and the game is not SOSP, a contradiction.

Last, consider the case $\left|P_{i}(h)\right|=2,{ }^{58}$ and let $P_{i}(h)=\{x, y\}$. Again by Lemma 5 , there can be at most one action $a \in A(h)$ such that $\left|P_{i}((h, a))\right|=2$. If there is no such action, then $h$ satisfies the requirements of a curated dictatorship. If there is such an action, it must be that $P_{i}((h, a))=\{x, y\}$ for some $a$, and $P_{i}\left(\left(h, a^{\prime}\right)\right)=\{x\}$ for all other $a^{\prime} \neq a,{ }^{59}$ which again satisfies the requirements of a curated dictatorship.

\footnotetext{
${ }^{57}$ Recall that $h$ is the first (and only) time $i$ is called to play in $\Gamma^{\prime}$, and so is on the path of play for all types.

${ }^{58}$ If $\left|P_{i}(h)\right|=1$, the argument is trivial.

${ }^{59}$ That is to say, all other actions must clinch the same payoff. If there were two actions $a^{\prime}, a^{\prime \prime}$ such that $P_{i}\left(\left(h, a^{\prime}\right)\right)=\{x\}$ and $P_{i}\left(\left(h, a^{\prime \prime}\right)\right)=\{y\}$, then it would not be strongly obviously dominant for any type to select $a$, and it can be pruned.
} 


\section{A.5 Proof of Theorem 6}

Suppose a game $\Gamma$ is strongly obviously strategy-proof, efficient, and satisfies equal treatment of equals. Our characterization of SOSP and efficient mechanisms tells us that we can assume that $\Gamma^{\prime}$ is equivalent to an almost-sequential dictatorship, which can be run as follows: at each history, including the empty history, Nature chooses an agent from among those agents who have yet to move, and this agent moves. If there are three or more objects or exactly one object still unallocated, then this agent selects his most preferred still available object and sends an additional message. If, for the first time, there are exactly two unallocated objects (and thus two agents yet to move), then the agent who moves either (i) selects his most preferred object and sends a message, or (ii) has a choice of clinching one of the two objects (and sending a message) or passing. In the latter case, Nature selects the other agent who has yet to move, who chooses his best object, and the agent who passed obtains the remaining object.

The reminder of the proof is by induction on the number $k$ of agents that have been called to move. We will show that each time Nature calls an agent to move, it must select uniformly at random from all agents who have yet to be called. Consider $k=1$ (i.e., the first agent called), and suppose there are more than three agents and objects. Consider a preference profile where all agents rank objects in the same order, $o_{1} \succ o_{2} \succ \cdots \succ o_{N}$. By equal treatment of equals, all agents must receive object $o_{1}$ with equal probability. Since, at this profile, $o_{1}$ is always taken by the first mover, we conclude that Nature must select each agent to be the first mover with equal probability.

Now, consider any $k$-th move, where $k<N$, and assume that for all moves $1, \ldots, k-1$, each remaining agent at that point was called on by Nature with equal probability. Label the history under consideration as $h$, and name the agents so that along the path to $h$, the first mover is $i_{1}$ who chooses $o_{1}$, then $i_{2}$ chooses $o_{2}$, etc. until $i_{k-1}$ chooses $o_{k-1}$.

Suppose first that there are at least three objects left. We claim that each agent who has not moved yet has an equal chance to be called by Nature at history $h$. To see why, consider some object $o \neq o_{1}, \ldots, o_{k-1}$ and the preference profile in which each agent $i_{\ell}$, for $\ell=1, \ldots, k-1$, ranks objects so that

$$
o_{1} \succ_{i_{\ell}} o_{2} \succ_{i_{\ell}} \ldots \succ_{i_{\ell}} o_{\ell} \succ_{i_{\ell}} o \succ_{i_{\ell}} \ldots
$$

and other objects are ranked below $o$. Let $Y(h)=I-\left\{i_{1}, \ldots, i_{k-1}\right\}$ be the set of agents who have yet to move at $h$, and assume that all $i \in Y(h)$ have the same preferences and rank $o$ first. The total probability that $i$ receives $o$ is equal to the sum of (1) the probability that Nature chooses $i$ at $h$ and (2) the probability that Nature chooses $i$ to move at any other 
$h^{\prime} \neq h$ where $o$ is still available. The crux of the argument is to note that for the preferences specified, for any other branch of the tree (that does not contain history $h$ ), object $o$ will be claimed by someone at the $(k-1)$-th move or earlier. By the inductive hypothesis, each time Nature picks an agent to move at any of these histories, all agents in $Y(h)$ have an equal probability of being picked, and, if they are, they will immediately claim object $o$. This implies that (2) is the same for all agents $i \in Y(h)$. Since the sum $(1)+(2)$ must also be the same for all agents (by equal treatment of equals), we conclude that (1) is also the same for all $i, j \in Y(h)$, i.e., the probability that $i$ is chosen to move at $h$ is equal to the probability that $j$ is chosen to move at $h$, as desired.

Last, suppose that there are exactly two objects $o$ and $o^{\prime}$ left at history $h$. With two objects, Bogomolnaia and Moulin (2001) show that Random Priority is the only strategyproof and efficient mechanism that satisfies equal treatment of equals, and the claim follows from their work. ${ }^{60}$

\section{B Extensions: Outside Options}

Consider the allocation model of Section 5, and suppose that each agent has an outside option.

\section{B.1 Individual Rationality}

We say that a game is individually rational if each agent can obtain at least his outside option. The analogues of our results hold true for individually rational games as soon as the domain of each agent's preferences satisfy the domain condition from Section 2 restricted to sets $X \subseteq \mathcal{X}$ that do not contain the outside option of this agent. Our proofs remain valid in this setting.

\section{B.2 Restricted Domains}

Our results hold true also in allocation domains in which any agent prefers any object to the outside option. Our proofs remain valid also in this setting. ${ }^{61}$

\footnotetext{
${ }^{60}$ Bogomolnaia and Moulin actually prove that Random Priority is the only strategy-proof and ordinally efficient mechanism that satisfies equal treatment of equals when there are three objects and three agents, and with two objects Pareto efficiency and ordinal efficiency become equivalent. The two object case is much simpler than the three object problem that Bogomolnaia and Moulin study, and one can easily verify the above claim without reliance on Bogomlnaia and Moulin's seminal analysis.

${ }^{61}$ Note that in this setting every game is individually rational, hence this observation is contained in the previous one.
} 


\section{References}

AbdulkadiroĞLu, A. And T. Sönmez (1998): "Random Serial Dictatorship and the Core from Random Endowments in House Allocation Problems," Econometrica, 66, 689-701.

(2003): "School Choice: A Mechanism Design Approach," American Economic Review, 93, 729-747.

Armstrong, M. (1996): "Multiproduct Nonlinear Pricing," Econometrica, 64, 51-76.

Arribillaga, R. P., J. Massó, And A. Neme (2017): "Not All Majority-based Social Choice Functions Are Obviously Strategy-proof," .

Arrow, K. J. (1963): Social Choice and Individual Values, New York: Wiley, 2nd edition ed.

Ashlagi, I. And Y. A. Gonczarowski (2016): "No Stable Matching Mechanism is Obviously Strategy-Proof." Working Paper.

Bade, S. And Y. Gonczarowski (2017): "Gibbard-Satterthwaite Success Stories and Obvious Strategyproofness," .

Bogomolnaia, A. And H. Moulin (2001): "A New Solution to the Random Assignment Problem," Journal of Economic Theory, 100, 295-328.

Budish, E. And E. CAntillon (2012): "The Multi-unit Assignment Problem: Theory and Evidence from Course Allocation at Harvard," American Economic Review, 102, 2237-71.

Chawla, S., J. D. Hartline, D. L. Malec, And B. Sivan (2010): "Multi-parameter mechanism design and sequential posted pricing," in Proceedings of the forty-second ACM symposium on Theory of computing, ACM, 311-320.

Che, Y.-K. And F. Kojima (2009): "Asymptotic Equivalence of Random Priority and Probabilistic Serial Mechainsms," Forthcoming, Econometrica.

(2010): "Asymptotic Equivalence of Random Priority and Probabilistic Serial Mechanisms," Econometrica, 78, 1625-1672.

Dur, U. M. And M. U. Ünver (2015): "Two-Sided Matching via Balanced Exchange: Tuition and Worker Exchanges," NC State University and Boston College, Working Paper.

Ehlers, L. (2002): "Coalitional Strategy-Proof House Allocation," Journal of Economic Theory, 105, 298-317.

Ehlers, L. AND T. Morrill (2017): “(Il) Legal Assignments in School Choice," .

Einav, L., C. Farronato, J. Levin, And N. Sundaresan (2018): "Auctions versus posted prices in online markets," Journal of Political Economy, 126, 178-215. 
Feldman, M., N. Gravin, And B. Lucier (2014): "Combinatorial auctions via posted prices," in Proceedings of the twenty-sixth annual ACM-SIAM symposium on Discrete algorithms, SIAM, 123-135.

GibBard, A. (1973): "Manipulation of Voting Schemes: A General Result," Econometrica, $41,587-601$.

(1977): "Manipulation of Schemes That Mix Voting with Chance," Econometrica, 45, 665-681.

Gul, F. And W. Pesendorfer (2001): "Temptation and Self-Control," Econometrica, 69, 1403-1435.

_ (2004): "Self-control and the theory of consumption," Econometrica, 72, 119-158.

Hagerty, K. M. And W. P. Rogerson (1987): "Robust Trading Mechanisms," Journal of Economic Theory, 42, 94-107.

Hakimov, R. And O. Kesten (2014): "The Equitable Top Trading Cycles Mechanism for School Choice," WZB and Carnegie Mellon University, Working Paper.

Hatfield, J. W. (2009): "Strategy-Proof, Efficient, and Nonbossy Quota Allocations," Social Choice and Welfare, 33 No. 3, 505-515.

Jehiel, P. (1995): "Limited Horizon Forecast in Repeated Alternate Games," Journal of Economic Theory, 67, 497-519.

_ (2001): "Limited Foresight May Force Cooperation," The Review of Economic Studies, 68, 369-391.

Jones, W. And A. Teytelboym (2016): "Choices, preferences and priorities in a matching system for refugees," Forced Migration Review.

KE, S. (2015): "Boundedly rational backward induction," Tech. rep., Mimeo, Princeton University.

KohlBerg, E. And J.-F. Mertens (1986): Econometrica, 54, 1003-1037.

Laibson, D. (1997): "Golden eggs and hyperbolic discounting," The Quarterly Journal of Economics, 112, 443-478.

LI, S. (2017): "Obviously Strategy-Proof Mechanisms," American Economic Review, 107, $3257-87$.

LiU, Q. And M. Pycia (2011): "Ordinal Efficiency, Fairness, and Incentives in Large Markets," Working Paper.

Loertscher, S. And L. M. Marx (2015): "Prior-Free Bayesian Optimal Double-Clock Auctions," Working Paper. 
MackenzIE, A. (2017): "A Revelation Principle for Obviously Strategy-proof Implementation," working paper.

Morrill, T. (2014): "Making Just School Assignments," Games and Economic Behavior, (forthcoming).

PÁpAi, S. (2000): "Strategyproof Assignment by Hierarchical Exchange," Econometrica, 68, $1403-1433$.

- (2001): "Strategyproof and Nonbossy Multiple Assignments," Journal of Public Economic Theory, 3, 257-271.

Pycia, M. (2011): "Ordinal Efficiency, Fairness, and Incentives in Large Multi-Unit-Demand Assignments," .

Pycia, M. And M. U. Ünver (2016): "Arrovian Efficiency in Allocation of Discrete Resources," UCLA and Boston College, Working Paper.

- (2017): "Incentive Compatible Allocation and Exchange of Discrete Resources," Theoretical Economics, 12, 287-329.

Rosenthal, R. W. (1981): "Games of perfect information, predatory pricing and the chain-store paradox," Journal of Economic theory, 25, 92-100.

Roth, A. (2015): "Migrants Aren't Widgets," Politico Europe, http://www.politico.eu/article/migrants-arent-widgets-europe-eu-migrant-refugeecrisis/.

Roth, A. E., T. Sönmez, And M. U. Ünver (2004): "Kidney Exchange," Quarterly Journal of Economics, 119, 457-488.

Satterthwaite, M. (1975): "Strategy-proofness and Arrow's Conditions: Existence and Correspondence Theorems for Voting Procedures and Social Welfare Functions," Journal of Economic Theory, 10, 187-216.

Shapley, L. AND H. SCARF (1974): "On Cores and Indivisibility," Journal of Mathematical Economics, 1, 23-37.

Sönmez, T. And M. U. Ünver (2010): "Course Bidding at Business Schools," International Economic Review, 51, 99-123.

Troyan, P. (2016): "Obviously Strategyproof Implementation of Top Trading Cycles," Working paper, University of Virginia.

Troyan, P., D. Delacretaz, and A. Kloosterman (2018): "Essentially Stable Matchings," working paper.

Zhang, L. And D. Levin (2017a): "Bounded Rationality and Robust Mechanism Design: An Axiomatic Approach," American Economic Review Papers and Proceedings, 107, 23539 . 
(2017b): "Partition Obvious Preference and Mechanism Design: Theory and Experiment," . 